\title{
A comparative study of time aggregation techniques in relation to power capacity expansion modeling
}

Buchholz, Stefanie; Gamst, Mette; Pisinger, David

Published in:

TOP

Link to article, DOI:

$10.1007 / \mathrm{s} 11750-019-00519-z$

Publication date:

2019

Document Version

Peer reviewed version

Link back to DTU Orbit

Citation $(A P A)$ :

Buchholz, S., Gamst, M., \& Pisinger, D. (2019). A comparative study of time aggregation techniques in relation to power capacity expansion modeling. TOP, 27(3), 353-405. https://doi.org/10.1007/s11750-019-00519-z

\section{General rights}

Copyright and moral rights for the publications made accessible in the public portal are retained by the authors and/or other copyright owners and it is a condition of accessing publications that users recognise and abide by the legal requirements associated with these rights.

- Users may download and print one copy of any publication from the public portal for the purpose of private study or research.

- You may not further distribute the material or use it for any profit-making activity or commercial gain

- You may freely distribute the URL identifying the publication in the public portal

If you believe that this document breaches copyright please contact us providing details, and we will remove access to the work immediately and investigate your claim. 


\title{
A Comparative Study of Time Aggregation Techniques in relation to Power Capacity-Expansion Modeling
}

\author{
Stefanie Buchholz • Mette Gamst · David Pisinger
}

Received: / Accepted:

\begin{abstract}
In this paper we study aggregation techniques for power capacity expansion problems. Combining a growing demand for green energy with a hard constraint on demand satisfaction causes system flexibility to be a major challenge in designing a stable energy system. In order to both determine the need for flexibility and which technologies that could satisfy these needs at minimum cost, the system should be analyzed on an hour-by-hour scale for a long period of time. This often leads to computationally intractable problems. One way of getting more tractable models is to aggregate the time domain. Many different aggregation techniques have been developed, all with a common goal of selecting representative time slices to be used instead of the full time scale, gaining a problem size reduction in the number of variables and/or constraints. The art of aggregation is to balance the computational difficulty against the solution quality, making validation of the techniques crucial. We propose new aggregation techniques and compare these to each other and to a selection of aggregation techniques from the literature. We validate the aggregated problems against the non-aggregated problems and look into the sensitivity of the performance of the aggregation techniques to different data sets and to the selection of different element types.

Our analysis shows that aggregation techniques can be used to achieve very good solutions in much shorter time, and that simple aggregation techniques achieve as good performance as techniques with higher complexity. Even though the aggregation techniques in this paper are applied to power capacity expansion models, the methodology can be used for other problems with similar time dependence, and we believe that results in agreement with the results seen here, would be achieved.
\end{abstract}

S. Buchholz

Technical University of Denmark,

DTU Management Engineering,

Produktionstorvet, 424, DK-2800 Kgs. Lyngby

E-mail: stebu@dtu.dk

M. Gamst

Energinet.dk,

Tonne Kjærsvej, 65, DK-7000 Fredericia

E-mail: mga@energinet.dk

D. Pisinger

Technical University of Denmark,

DTU Management Engineering,

Produktionstorvet, 424, DK-2800 Kgs. Lyngby

E-mail: dapi@dtu.dk 
Keywords Domain reduction, time aggregation, power system planning, capacity expansion, solution time and quality balance, validation.

\section{Introduction}

With the power generation mix being increasingly dominated by Variable Renewable Energy (VRE) sources such as Photovoltaics (PV) and wind [4,26], the variability, and by that also the uncertainty, is increasing, causing operational challenges in the power systems [1]. A core priority is to achieve high stability in the systems. Stability of an energy system can be secured by including fast responding resources, counteracting the load variability. The need for such resources is referred to as the need for flexibility [6]. Today, the flexibility used to stabilize energy systems is dominated by power plants based on fossil fuels, but moving toward low-carbon economies, the need for low-carbon flexibility options rises. Increasing the VRE shares complicates the quantification of the flexibility need, asking for more advanced models that simultaneously analyze short term-system operations and long-term investment decisions [9]. Both the determination of the need for flexibility as well as the optimal flexibility technologies satisfying these needs at minimum overall cost, have to be analyzed on an hour-by-hour scale for a long period of time. One approach is to combine a short term dispatch model containing Unit Commitment (UC), with a long term investment model. The resulting model is referred to as the Capacity Expansion Model with Unit Commitment (CEMUC). With high computational difficulty in both the short term and in the long term models, the combination of the two may lead to computationally intractable models.

A common approach to handle this high level of computational difficulty is to reduce the time domain. The aim is to select representative time slices that simultaneously reflect the annual variability of demand, wind and solar resources, as well as the correlation between them. With the goal of reducing the problem size, numerous aggregation techniques have been defined. The general idea of aggregation is to group elements of a reference time series and select a representative for each group. Replacing a group of elements with a single element causes a reduction in the number of variables leading to a more computationally tractable problem. The base of an aggregation technique is the decision of how the data is grouped. The answer to that question categorizes the different aggregation techniques, but to specify the single aggregation, a number of other questions need to be answered as well: What data to group? What defines a group element? How to select a representative from each group? For aggregation techniques not pursuing time chronology, how to order the selected elements? With a variety of answers to each question, it is no surprise that so many different single aggregations have been developed over time. The art of aggregation is to balance the computational difficulty against the solution quality. Consequently, the validation of aggregation techniques is crucial, but yet this still seems to be a lacking part of the literature. Validation can be categorized as data or model validation. Although the aggregation methodology spans from heuristics to optimization theory, and even covers machine learning techniques, the common focus is to reduce a large set of data to a more tractable size. Therefore, the probably most intuitive and also the most used validation is measuring the ability of the aggregated time series to replicate the original one. This is denoted data validation, while model validation is to analyze the ability of the aggregated problem to replicate investments achieved by the original problem. An interesting question, which to our knowledge, is not yet addressed in the literature, is whether a good performance according to the commonly used data validation also ensures good performance in a model validation? To answer this question, we define a small computational tractable CEMUC and using various aggregation techniques, which we believe to have scalable performance, we derive aggregated problem instances, which we then validate according to the original problem.

Closing another gap in the literature, aggregated problems will not only be compared to non-aggregated problems but also to each other. Furthermore, the sensitivity of the performance of the aggregation techniques to different data sets is analysed. A common disadvantage of aggregation techniques in the literature is smoothing of the supply profiles which causes an underestimation of the need for flexibility [1,32]. We 
propose a few new aggregation techniques, and, with a focus on the complexity of the aggregation techniques, we analyze whether more complex aggregation techniques actually provide better estimates of the needed flexibility.

Herewith, the main contributions of this paper is an overview and comparison of different aggregation techniques and an analysis of aggregation complexity gains. Furthermore, we contribute with a suggestion of four new aggregation techniques, as well as a systematic way of testing aggregated instances in a capacity expansion modelling framework. A last contribution is a comparison of the suggested model validation to one of the widely used data validations.

The terminology used in this paper is described in Figure 1. We refer to data sets as Instances (or commonly time series) and the methods we use to reduce these are called Aggregation Techniques. Whenever an instance has been reduced (aggregated) we refer to it as an Aggregated Instance, while non-aggregated data sets are referred to as Original Instances. The MIP model taking the instances as input are simply referred to as Model, and whenever this model is applied to the aggregated and the original instances we refer to the resulting problems as Aggregated Problem (Instance) and Original Problem (Instance), respectively. A description of the abbreviations used throughout the paper can be seen in Appendix A.

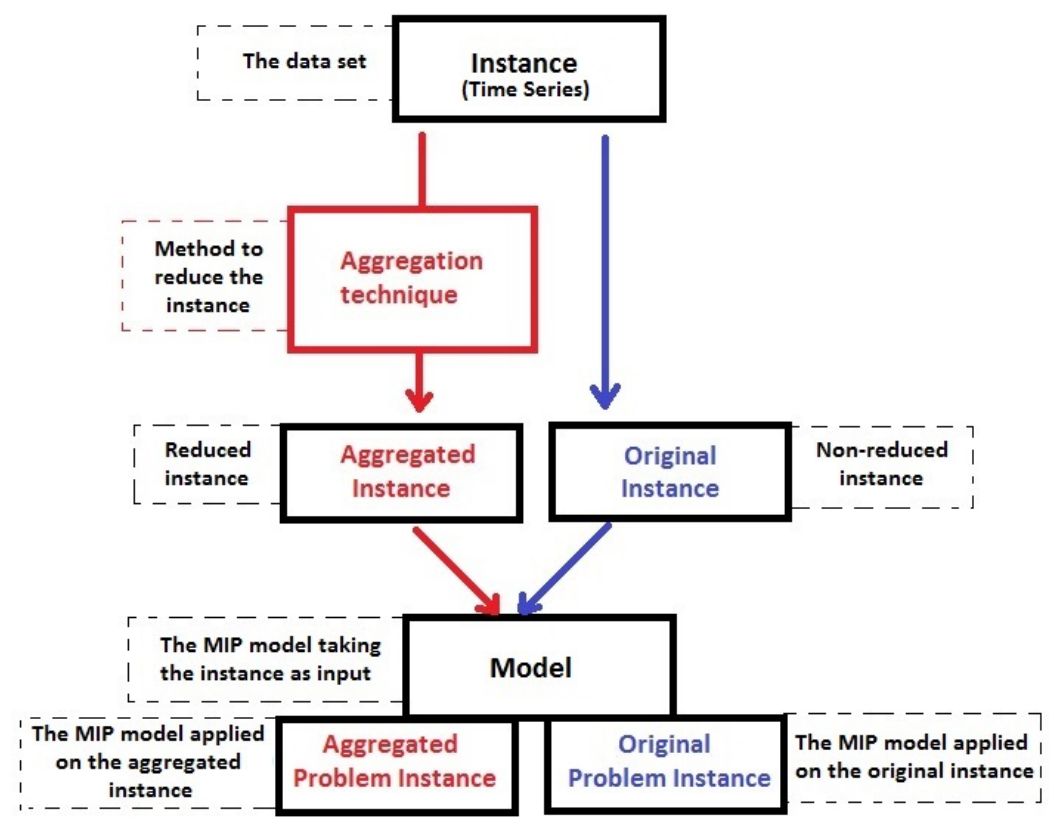

Fig. 1: Terminology used in this paper

The remainder of this paper is structured as follows; Section 2 provides a literature review of aggregation techniques while Section 3 introduces the proposed new aggregation techniques and defines the different aggregation strategies analyzed in this paper. Section 4 covers data and model descriptions while the systematic validation method is proposed in Section 5. Section 6 provides the results of the case study as well as a discussion about what we can learn from the experiments. All observations are concluded in Section 7 . 


\section{Literature Review}

Aggregation techniques can be divided into 3 categories, namely Heuristic Selection (HS), Cluster Analysis (CA) and Optimization (OPT). Techniques of the first two categories consist of selecting time slices according to some predefined rules, and then validate the resulting time series according to chosen criteria. Contrary, OPT-based techniques find the aggregated time series as the best selection according to predefined criteria.

\subsection{HS based Techniques}

HS approaches are the most simple and intuitive. The idea is to identify typical behavior of the original time series, and select time slices representing these behaviors. Many HS variations exist, but frequently historical weeks, days or hours are selected according to seasonality, weekend/workday, night/day and peak/off-peak deviations. In [1], a workday and a weekend-day for each of the 4 seasons are selected, both represented by four 6 -hour block averages. The result is a 32-time-sliced aggregated time series, which the authors conclude to represent the seasonal demand variation well. Other HS based aggregations are summarized in Table 21 in Appendix B and furthermore in [23]. Many of these aggregation techniques have the drawback that they, like the technique in [1], are developed only for capturing the variability in demand. With VRE getting increasing shares of the energy production, there is an increasing focus on also capturing the variability in the supply side. In [21], it is concluded that aggregation techniques developed with only demand variability in focus, need many more time elements to also represent the variability in supply. The challenge lies in representing the variability of wind, PV and demand in combination as seen in [18], where the effect of grouping 8760 hours into blocks of 2, 6, 12 and 24 consecutive hours, represented by their average value, are tested. Through a model validation, it is concluded that while the variability of demand and PV are well caught, the wind shows strongly stochastic properties, causing it to be difficult to represent by averaged time slices. The research in [24] concludes that the more wind, the larger errors are observed when time is aggregated, which typically is seen as missing investments in base-load technologies.

To account for the increased variability caused by VRE, the HS approaches are modified with the aim of putting more focus on the extreme cases of the variability. In [13], 3 days from each season are selected, representing the low, medium and high VRE supply regimes. Furthermore, a super peak element, representing high demand and low VRE supply, is added. To minimize the smoothing effect on the variability, [30] suggests to select the most variable wind profile for each day type of each season. However, no analysis of the benefit of this is provided since the original model is intractable by state of the art solvers.

\subsection{CA based Techniques}

Alternatively, aggregation approaches based on CA typically have a better and a more automatic capturing of peak groups. A specific CA approach is defined by the way elements are grouped into clusters. In general, the aim is to minimize the similarity between clusters while maximizing the similarity within each cluster. The similarity metric is frequently defined such that the objective is to minimize the within cluster variance or to minimize the mean distance between the elements in a cluster and the centroid of that cluster [29]. When each element is assigned to only one cluster, the technique is referred to as Exclusive, while overlapping cluster techniques assign each element to all clusters associated with a degree of membership. In relation to time aggregation, exclusive $\mathrm{CA}$ techniques are the most used and these are further classified into Hierarchical and Partitioning Clustering ( $\mathrm{HC}$ and PC). For the first category the aim is to build a hierarchy of clusters with a sequence of nested partitions. Either each element is assigned its own cluster and then clusters are merged until a single cluster is achieved (Agglomerative clustering), or all elements are 
assigned to the same cluster and the cluster is then divided until each element has its own cluster (Divisive clustering). The resulting hierarchy tree is cut at the wished number of clusters. PC selects a pre-defined number of elements to be centroids, and then new centroids are calculated as the rest of the elements are allocated in the clusters. This is repeated until no further improvements (according to the similarity metric) are seen. Papers studying CA based aggregation techniques are listed in Table 22 in Appendix B.

\subsection{Element Selection from Groups}

After the clustering, representative elements from each cluster need to be selected and weighted such that the aggregation reflects the relative importance of the elements in the original problem. As soon as groups have been formed, the selection methodology is the same for CA techniques and for group-based HS techniques. Various methods are seen in the literature, and [12] gives a good overview of some of these. The probably most intuitive and applied technique is to replace the group with an average of the group elements. This is referred to as the Integral Method [18] and it is widely used but also criticized due to peaks being smoothened [24,27]. In general, aggregation based on averaging often leads to an underestimation of production and of the need for storage capacity and storage technologies, leading to an underestimation of the overall system costs [1]. Alternatively, [12] suggests choosing the day closest to the centroid of the cluster, but argues that this day likely would be an average day, and consequently, also represent a smoothened profile. Furthermore, they suggest selecting a single random day, but with the risk of not capturing the total level of demand. Another suggestion is to reduce the cluster sizes by disregarding days which deviate significantly from the cluster's average, but this shows worse results compared to keeping all information in the clusters [12]. Regarding weightings, a fixed weighting is often seen, where either information from each cluster is assumed to be equally important, be important proportionally to the size of the cluster, or the weighting could choose only to represent a partition of the clusters [29]. To our knowledge, there is no clear conclusion regarding the exsistence of a single best selection criteria nor a single best weighting strategy. In [20], aggregated time series are found using a $k$-means-, a hierarchical- and a fuzzy clustering approach and they are compared according to a selection of mean and median representatives. Through a data validation, using a defined performance index, the $k$-means approach with median element selection is concluded to provide the best results for any number of clusters. However, a clear conclusion of the median element, in general, being the best, is not drawn.

\subsection{Challenges of the Approaches}

Even with CA approaches being better at capturing variability in supply and demand, still many of the approaches in the literature are developed only for capturing the variability in PV and demand. Consequently, also for these approaches, unwanted properties such as smoothing of the profiles and underestimation of flexibility are seen [21]. To minimize the smoothing effect of averaging clusters, [12] suggests averaging the days, both according to level of demand and to hour-by-hour changes in demand. That is, if the majority of elements in a cluster have increasing demand, then the resulting cluster profile should increase with the average of the total cluster increase. To sufficiently reflect the characteristic fluctuations of both demand and VRE availability, [23] presents a multi-dimensional algorithm based on hierarchical clustering, which allocates every historical day into clusters, such that the inner cluster variance is minimized. From each cluster, the day closest to the mean value of the cluster is chosen.

An aggregated time series that captures annual electricity demand and average VRE capacity factors for each region, region specific Load Duration Curves (LDCs) of electricity demand and VRE technologies, and spatial and temporal correlation of electricity demand and VRE electricity infeed are, by [23], defined to replicate the variability of both demand and VRE. In addition, [22] identifies the core of any method 
assessing resource demand, as identifying periods with high risk of not meeting demand. As a consequence of VRE being uncertain, the interesting point is to identify the periods where the VRE does not meet demand or produces too much energy. Therefore an appealing measure in the development of time aggregation techniques is the Residual Load Duration Curve (RLDC) which is defined as the power generated from VRE, subtracted from the power demand in each time point, and then sorted in decreasing order [32]. The positive area below this curve is the demand not satisfied by the VRE-based power, while the negative area is the overproduction of VRE power. According to [32], the RLDC does not only illustrate the challenges of VRE but also quantifies these regarding underestimation of base load capacity, overproduction of VRE and reduced full-load hours. Using a time resolution with a too flat RLDC approximation, causes over- and underproduction of VRE to be underestimated [27]. Consequently, the solutions will not reflect the need for flexibility such as the need for technologies handling shut downs or the need for storage or back-up productions.

\subsection{OPT based Techniques}

To secure the best approximation of the RLDC, an idea is to select the time elements such that the difference between the RLDCs are minimized, instead of only using the RLDC difference as a post-validation. This is a typical objective of the optimization based aggregation techniques. Generally, using optimization to find aggregated time series is often more challenging regarding implementation and these approaches are typically more time consuming compared to the HS and CA approaches.

The work of [6] proposes a method to optimally select a predefined number of representative historical weeks that jointly represent the demand and VRE output for capacity planning models aimed at evaluating the need for flexibility. An exhaustive search is used to find the selection of historical weeks minimizing the sum of squared errors between the original and the approximated RLDC, as suggested by [23]. The same approach is used in [7] to select 4 historical weeks. Since the weeks are found using an exhaustive search, the predefined number of weeks has a high influence on the solution time. For example, as stated in [6], finding one week takes $0.05 \mathrm{sec}$, finding 4 weeks takes $10 \mathrm{~min}, 8$ weeks takes 19 days while finding 13 weeks takes approximately 46 years. Despite the computational difficulty, [7] concludes that the 4 week selection closely matches the shape of the original RLDC, that the approach shows better results compared to other approaches, and that it is robust to the choice of model.

In [28], an optimization model is used to select representative days with the aim of minimizing the difference between the original DCs and the DCs of the selected days. To represent the DCs in the optimization framework, these are approximated using an equal sized bin representation. Consequently, the quality of the aggregation is limited by the quality of this approximation. In [33], it is suggested that the RLDC is approximated using several squares and a triangle, and then that the evolution of the RLDC is parametrized such that the shapes of the squares and triangle dynamically change depending on the modelendogenous share and mix of VRE. This is concluded to be a more accurate approximation compared to the bin representation, however it has the drawback of not being compatible for models having multiple regions. In addition, constructing RLDCs has the drawback that the correlation between the single profiles is lost and consequently that a good approximation of the RLDC does not ensure a good representation of the single demand, wind and PV curves [27]. This causes an extension of the work in [28], where a RLDC is introduced for each time series and a Correlation Duration Curve (CDC) is approximated for each aggregated time series. Unfortunately, they do not evaluate the performance of this technique, which actually is very common in the time aggregation literature. 
2.6 Importance of Validation

Based on the presented literature, it is clear that the time domain reduction methodology provides simplification techniques enabling a reduction of the problem sizes which commonly leads to reduced computation times. To highlight some of the achievements, we mention [30], where a spatio-temporal MILP, based on a 2160 hour resolution is shown to be computationally intractable. With an HS aggregation based on a dynamic block structure, representative time series are found with resolutions of 720, 168 and 24 time slices. These aggregations make it possible to achieve solution times of $833 \mathrm{~min}, 679 \mathrm{sec}$ and 18 sec respectively. Furthermore, in [22], aggregation techniques reduced solution times from 2 hours to 185 sec, while in [12] a reduction from $22 \mathrm{~min}$ to $26 \mathrm{sec}$ was seen. In [30], the authors show that, while the problem size seems to increase linearly with the length of the planning horizon, the solution times seem to increase exponentially, which clearly demonstrates the benefit of finding a reduced time resolution. However, with an aggregated problem only being an approximation of the original problem, achieving same accuracy cannot be expected. Consequently, validation of the aggregated problem solutions is crucial. Nevertheless, as pointed out in [23], generally validation is a lacking part of the literature as seen in e.g. [13,30,12]. Consequently, much aggregation is made without any published knowledge about the quality of the resulting selection. An obvious reason for this is that aggregation often is used as a necessity due to computationally intractable problems [30], which also makes validation difficult. Most of the highlighted literature in this review comes with some sort of validation, and an overview of examples is provided in Table 23 in Appendix B.

Most commonly, aggregated time series are validated according to their ability of replicating the original RLDC, as seen in [24] and [1]. In addition, some studies replace or add to the data validation an analysis of the accuracy of the aggregated problems $([24,22,21])$. Typically, this includes comparing solutions achieved from solving original problems to solutions achieved from solving aggregated problems. However, this is only possible if the original problems are tractable. In [3], they suggest a validation method assuming the original solution to be unknown and hence it is based on some sort of approximation of this. In [13] and [23], problem solutions for different resolutions are studied and low resolution solutions are evaluated against solutions based on higher resolutions to provide some validation of the number of elements included in the aggregations. However, the original resolution is not included in the study. Similarly, an example of data validation used to identify the amount of elements needed for a good representation of the original data is seen in [21]. Comparing solutions of original and aggregated problems is done in many ways. While [24] compares on investment decisions, VRE curtailment and market value of VRE, [12] compares on average and marginal costs and carbon, and on gas and coal output and profit. [21] looks at differences in system costs while [6] evaluates on energy contribution, capacity factors and the number of start-ups. In [28], the focus is on installed capacity and annual operational costs. From the different examples one can derive that installed capacity and system cost seems to be common comparison factors, however there is no clear suggestion of how to compare the solutions.

The reduction in computational difficulty depends partly on the number of elements selected and possibly also on the type of elements. Within the time aggregation framework, the element types to allocate are typically weeks, days or individual hours. The suitable number of groups to generate is problem and purpose dependent, and therefore no theoretically correct value exists. Within CA theory there are some methods to find an optimal amount of clusters, and even to find the optimal choice of clustering algorithm [15]. However, these methods require the data to show some cluster tendency, which cannot be guaranteed for residual loads. For several of the mentioned studies, the number of groups is tested in the light of balancing solution quality and solution resources. In [12], aggregated time series of 10 days show good results while [23] concludes that only 6 days are needed for their problem. According to [17], energy models covering one year, are well represented by 30 days corresponding to approximately 4 weeks or 672 hours. 


\subsection{Comparison of Aggregation Techniques}

Whenever aggregated problems can be validated, it opens up for the possibility of comparing different aggregation techniques. However, despite many different aggregation techniques being suggested in the literature, the comparison between different techniques is only rarely studied. Comparison of aggregation within the same category is seen in [17] where a $k$-means clustering, a HC and a combination of the two are compared. In [20] a $k$-mean clustering is compared to a HC and a fuzzy clustering approach. The authors of [21] compare different HS-based selections, though more with a focus on the size of the aggregated time series. In [27], four HS aggregations based on seasonal day and VRE level selections, considering both average days and representative days, are compared and validated according to their ability of replicating the original RLDC. The validation of the different techniques are performed for a varying penetration of VRE. They conclude that accounting for VRE levels in the selected elements showed good results, but since chronology was not maintained, it is less suitable whenever the potential of specific flexibility options needs to be analyzed. Selecting representative days results in intra-daily chronology being maintained, but it requires a larger number of time slices to achieve the same level of VRE capture as is captured when elements are selected according to VRE level.

Comparisons across different types of aggregation techniques are more rarely seen, only two cases have come to our attention. In [22] a $k$-mean clustering is compared to an HS. It is concluded that, unless a special peak modification is performed in the CA technique, the HS approach needs to select less days compared to the clustering to reach a specific performance. Secondly, the authors of [28] compare their own optimization approach based on a MILP to techniques based on heuristics, Ward's hierarchical clustering algorithm and Random selection. Also a Hybrid approach using random selection followed by optimal weighting is included in the comparison. The approaches are validated according to how well the capacity mix is captured in an energy model and they conclude that the MILP approach showed the best results regarding solution quality. However, it was very time consuming; only a selection of 2 days was possible within a timeframe of 6 hours CPU time.

\section{Aggregation Techniques}

With the intention of covering a variety of different techniques, we identify a set of representative Aggregation Strategies from each of the categories identified in Section 2. We ensure different complexity levels among the selected techniques. We also propose four new aggregation techniques, which we later compare to each other and to the techniques chosen from the literature. Inspired by the conclusions of [17], original time series cover 1 year of residual load data while all aggregated time series consist of 4 weeks/28 days/672 residual load hours, corresponding to a $92 \%$ reduction in data. Optimal selection of element type, such as hours, days or weeks, appears to be an open question in the literature. Only isolated conclusions about well functioning element types are made [1]. In this paper we compare the effect of choosing weeks, days and hours for the different aggregation techniques, with the goal of identifying a single best element type. Lastly we categorize techniques into Grouping-Strategies (GS) and Non-Grouping-Strategies (NGS). The first category includes techniques, which first group data and then select representatives from each group. The second category includes techniques, which select representatives right away without any grouping.

In the following we list the selected strategies and provide a brief description of each. We state the category, the suitable element types and the source of the technique.

$\rightsquigarrow$ Dummy selection (DX) (Our suggestion - HS category)

A very simple aggregation approach is included in the comparison, to motivate the analysis of the relation between complexity and performance of aggregation strategies. The probably most simple HS is the random selection, but in order to avoid the randomness we instead perform the selection of every 
$13^{\text {th }}$ element of the residual load time series. The chronology of the selected elements is respecting the time chronology of the original series. The performance of this aggregation strategy is expected to be very data dependent, but since some seasonality is seen in the original profiles, this approach could still be valid.

$\rightsquigarrow$ Statistical Representation (SR) (Based on [22] - HS category)

The approach aims at selecting elements such that the resulting aggregated time series have mean values and standard deviations as close to the values of the original time series as possible. Furthermore, high correlation between the aggregated time series and the original time series is aimed at as well. Practically, 10.000 random samples of the elements are constructed, the statistical measures for each sample is calculated, and then the sample whose statistical measures most closely resembles those of the original time series, is selected as the aggregated time series. The chronology of the selected elements is respecting the time chronology of the original series.

$\rightsquigarrow$ RLDC Selection (RL) (Based on [16] - HS category)

The proposed aggregation technique is one of the more complex HS aggregations and it shows good results in [16], which makes it interesting for our comparison. In contrast to techniques selecting historical elements, this aggregation technique constructs the elements by selecting the most interesting historical hours. The original Residual Load Curve (RLC) is divided into $\mathbb{X}$ parts, and for each part the RLDC is constructed and every $13^{t h}$ hour is selected with the first and the last hour included. By that, minimum and maximum levels of each part of the time series are selected, and by choosing every $13^{t h}$ hour, one hopes to capture all the different levels of the yearly RLDC. Since the selected hours do not have a natural chronology, this is reconstructed by choosing a chronology of one of the elements from each sample period. The most suitable chronology is chosen based on a RLDC and a Ramping Duration Curve (RDC) comparison. We look at 3 different variations of this approach where $\mathbb{X}$ is chosen as 1,4 and 28 , representing an hourly, a weekly and a daily perspective. For $\mathbb{X}=1$, every $13^{\text {th }}$ hour is selected from the full RLDC, giving an 672-hour selection, and the chronology is chosen as the time chronology of the selected hours. Choosing $\mathbb{X}=4$, every $13^{\text {th }}$ hour of each seasonal RLDC is selected, resulting in four 168-hour selections, and the chronology for each is chosen according to a selected week in the corresponding seasonal period. Lastly, $\mathbb{X}=28$ divides the RLC into 28 pieces and selecting every $13^{\text {th }}$ hour of the corresponding RLDCs, results in 28 24-hour selections. Each selection gets its chronology from a selected day in the corresponding RLC part. Essentially, this approach constructs days and weeks from hourly selections, and consequently, the daily and weekly perspectives are not fully comparable to other selections of days and weeks.

$\rightsquigarrow$ Dynamic Blocking (DB) (Our suggestion - HS category)

To better analyze the effect of time chronology, we suggest dividing the original time series into blocks, containing similar elements, while time chronology is respected. In [18], an equal sized block structure is suggested where the mean of each block is used as a representative. We suggest having a dynamic block structure, where a chosen criteria decides whether we move to the next block or not. In that way, we make sure, not to split similar elements into different blocks. The criteria used for deciding memberships of the different elements should ensure similarity within the blocks. Many different criteria are possible and the choice of criterion can have high influence on the performance of the aggregation strategy. We define our criterion to include an element $\left(E_{t}\right)$, if the inclusion does not cause the difference between the minimum and maximum element of the block $\left(B_{i}\right)$ to exceed a limit $\mathbb{Y}^{1}$. In mathematical terms this

\footnotetext{
1 Initially we included an element in the block, if the inclusion did not change the mean of the block with more than a value $\mathbb{Y}$. However, this had the disadvantage that very different elements potentially were grouped together if a slow decreasing/increasing period of elements was located between them.
} 
can be written as

$$
\begin{cases}E_{t} \in B_{i} & \text { if } \quad\left|\max \left(B_{i} \cup E_{t}\right)-\min \left(B_{i} \cup E_{t}\right)\right|<\mathbb{Y} \\ E_{t} \in B_{i+1} & \text { otherwise, }\end{cases}
$$

where the maximum limit $\mathbb{Y}$ is chosen either such that a predefined number of blocks is achieved or such that a target similarity is achieved within the blocks. The defined criterion works well for a dynamic blocking where single hours are selected. In that case, the maximum and minimum element is defined as the hour having maximum/minimum residual load value. Whenever we have an element defined by consecutive hours $|\mathrm{h}|$, the definition of maximum and minimum elements is not as straightforward. Also, for elements with a duration longer than one hour, similarity can be measured on the hour-by-hour change within an element. Consequently, when selecting weeks or days we define the blocking criterion to be a weighted term taking both variance, actual values and correlations into account. We define an element $\left(E_{t}\right)$ to be similar to elements of a block $\left(B_{i}\right)$ if the variance is close to the average element variance in the block, if the element level sum is close to the average level sum of the block and if the average correlation of the element with the rest of the elements is close to 1 . Due to the three different criteria having different magnitudes, we apply a weighting to make each criteria contributing significantly to the resulting multi-criteria term. In mathematical terms, we have,

$$
\begin{cases}E_{t} \in B_{i} & \text { if } \quad \text { CheckPoint }<\mathbb{Y} \\ E_{t} \in B_{i+1} & \text { otherwise }\end{cases}
$$

where the multi-criteria checkpoint is defined as follows

$$
\text { CheckPoint } \left.=W_{1}\left(\overline{\sigma_{B_{i}}^{2}}-\sigma_{E_{t}}^{2}\right)+W_{2} \mid \overline{\sum_{|h|} B_{i}}-\sum_{|h|} E_{t}\right) \mid+W_{3}\left(1-\overline{\sigma_{B_{i}, E_{t}}^{2}}\right)
$$

Here, the variance of an element and the average block variance is defined as

$$
\sigma_{E_{t}}^{2}=\frac{1}{|h|} \sum_{i=1}^{|h|}\left(e_{i}-\bar{e}\right)^{2}, \quad \sigma_{B_{i}, E_{t}}^{2}=\frac{1}{\left|B_{i}\right|} \sum_{E_{t} \in B_{i}} \sigma_{B_{i}, E_{t}}^{2}
$$

where $|h|$ refers to the amount of hours in the element, $e_{i}$ is the residual load of the $i^{t h}$ hour of the element, $\bar{e}$ is the average residual load value over all hours of the element and $\left|B_{i}\right|$ refers to the amount of elements $E_{i}$ in the block $B_{i}$. The value of $\mathbb{Y}$ is chosen such that either 4 blocks (week selection) or 28 blocks (day selection) are constructed. Since this approach is a $G S$, the blocking is followed by a selection as will be described in Section 3.1. The chronology of the selected elements is respecting the time chronology of the original time series.

$\rightsquigarrow$ Single Cluster (SC) (Based on [20] - CA category)

The suggested aggregation is a simple clustering algorithm based on the $k$-means clustering. In [20], the approach is compared to a hierarchical and a fuzzy clustering, and it is concluded to perform better, which is why it is selected for our comparison. The elements to be clustered are either single hours, days or weeks and the number of clusters to be generated is then 672, 28 and 4 respectively. After the clustering, elements from each cluster are selected (Section 3.1), and this selection then form the aggregated time series. As described in Section 2, the $k$-means clustering belongs to the PC family and hence it starts out by randomly selecting a set of elements to be group centers. Afterwards, elements are assigned to each group, such that the sum of squared differences between elements and the assigned cluster centers are minimized. We refer to [14] for further details about the general algorithm. The 
randomness of the initial choice of centers can affect the performance of the algorithm and to regain some stability it is recommended to evaluate the algorithm for multiple sets of initial center elements, and then to use the resulting best clustering. We run the algorithm for 25 initial sets of element centers. When days and weeks are clustered, the chronology of the selected elements is found as the ordering of the clusters according to the included hours. When hours are clustered, the chronology of the aggregated time series is chosen as the time chronology of the selected hours.

$\rightsquigarrow$ Cluster-Cluster (CC) (Based on [17] - CA category)

The idea is to combine a $k$-means clustering with a hierarchical clustering in a two-stage clustering procedure. First, $\mathbb{X}$ clusters are found using a standard $k$-means clustering, and then each of these clusters is divided into $\mathbb{Z}$ sub-clusters using a suggestion of an agglomerative HC clustering, based on a minmax linkage criterion and a dynamic time wrapping distance matrix. We refer to [17] for details about the specific algorithm. Essentially, the benefit of using a dynamic time wrapping distance matrix is, that this accounts for time series not being perfectly aligned or having different shape properties. An example could be offsets in loads due to daylight-savings. Since our time aggregations only consider a single RLDC, we use a normal euclidean distance instead of the time wrapping distance matrix. To further simplify, we use the complete linkage criterion rather than the minmax linkage. Algorithmically, this means that the distance between two clusters is measured as the distance between the elements from each cluster that are furthest apart, rather than measuring the distances as the radius of the smallest enclosing ball, centered at a point chosen from the two clusters. Again, 25 initial sets are used for the $k$-means clustering.

Clustering hours, we initially perform 224 clusters and then find 3 sub-clusters for each, resulting in 672 clusters. For day and week clustering, initially 2 and 7 clusters are created with each being divided into 2 and 4 clusters respectively. An element from each cluster is selected according to the selection criteria defined in Section 3.1. The chronology of the hour selection is chosen as the time chronology of the selected hours, while the chronology of the day and week selections is decided from the ordering of the clusters according to included elements.

$\rightsquigarrow$ Level-Correlation-Clustering (LC) (Our suggestion - CA category)

The aim of any clustering algorithm is to achieve as much similarity within each cluster and as little similarity between clusters as possible. Typically, the similarity measure is chosen to either be similarity in value of the clustered elements or similarity in the behavior of the clustered elements. In a time aggregation domain, this can be elements with similar levels being clustered together or elements with similar evolution in time being clustered together. Typically, this preserves either the replication of the RLDC or the replication of the RDC. According to [28], it is important to capture both, and hence we suggest combining cluster techniques pursuing both objectives.

Initially, we perform a fuzzy clustering in order to group elements according to their similarity in level. The fuzzy clustering is chosen since we need each group to have a minimum amount of members in order to enable multiple clustering. Therefore, if any group, after the first clustering, contains fewer members than needed, the fuzzy clustering provides a degree of membership for each element to each group, enabling a fast identification of members being suitable for the low-member groups. By this, our manipulation of the groups has few consequences. In order to ensure selection from each cluster with different behaviors, each cluster is re-clustered according to group members with similar behavior. For this, a hierarchical clustering is used, where the dissimilarity between two elements $i$ and $j$ is defined as

$$
d_{i j}=1-\rho_{i j},
$$

where $\rho_{i j}$ is the correlation between the two elements. This ensures that very similar elements get a small distance from each other and, therefore, likely get assigned to the same cluster, and vice versa. Selecting precisely one element from each of the resulting clusters ensures that the selection includes elements with different behavior for different levels. Of the considered element types in this paper, days is the most suitable for this approach. When clustering days, initially 7 clusters are constructed and then 
each is divided into 4 sub-clusters. When weeks are to be selected, initially two clusters are constructed and each is then divided further into two sub-clusters. As a consequence of the very low amount of clusters, this approach will most likely be similar to a single clustering with 4 initial clusters. Due to the consideration of correlation between elements, single hours are not suitable elements. We therefore perform an alternative nested clustering for this element type, where we assume some daily behavior correlation by initially clustering days. The idea is to cluster all days of a year into 112 clusters, and then all hours within each cluster are further clustered into 6 clusters. The outcome is 672 clusters covering different amounts of hours. The resulting aggregated time series is achieved by selecting one hour from each cluster according to the selection criteria defined in Section 3.1. This approach combines the selection of days and hours but in the end a single hour is selected, which categorizes it as an hour selection approach.

$\rightsquigarrow$ Exhaustive Search (ES) (Based on [5] - OPT category)

The suggested aggregation selects the 4 historical weeks that best represent the yearly RLDC. Using 4 nested loops, every combination of a 4 week selection is tested. For each selection, the RLDC is constructed and a Normalized Root Mean Square Error (NRMSE) is found between this approximated RLDC and the original RLDC. The 4 week selection having a RLDC closest to the original RLDC forms the aggregated time series. Even though, theoretically, this approach could be performed for any type of element, only the selection of weeks will be practically suitable. The smaller the elements are, in terms of time duration, the more combinations need to be checked. The complexity of the algorithm can be expressed as $O\left(\frac{(|E|) !}{(|E|-|C|) !}\right)$, where $E$ is the number of elements and $C$ is the cluster size. Consequently, to find a selection of 4 weeks, $\frac{52 !}{48 !}=6.497 .400$ combinations need to be checked which makes it challenging to perform larger selections. A 28 day selection requires $1,8 \cdot 10^{71}$ combinations to be checked while a selection of 672 single hours requires an uncountable amount of combinations to be checked.

$\rightsquigarrow$ Optimized RLDC Approximation (OA) (Based on [28] - OPT category)

As described in Section 2, the suggested approach approximates the original RLDC using an equal sized bin structure and then finds an optimal selection of days, which has the approximated RLDC closest to the original one. The original RLDC is divided into a fixed amount of bins and then for each element of the year, its share of time within each bin is calculated. Afterwards, an optimization model finds the selection of elements which has a share of time within each bin distribution as close as possible to the distribution of the original time series. Essentially, the idea is the same as in the ES approach, but instead of trying out all combination the selection is found by using state of the art solvers, minimizing the difference between the RLDCs. Only the selection of days will be considered in this approach.

$\rightsquigarrow$ Optimized criteria Selection (OS) (Our suggestion - OPT category)

Inspired by the SR approach in [22], we suggest an aggregation technique based on the same idea but in an optimization framework. The idea is to choose elements such that the correlation between selected elements is minimized and values as close as possible to the average element variance and the overall average, maximum and minimum levels and rampings of the original time series are achieved. Applying a weight to the different statistical goals and adding the terms results in a multi-objective function to be minimized. Due to this approach considering variation and correlation of elements, only days and weeks will be considered. The mathematical formulation is described by equations (1)-(24). Sets, parameters and variables are listed in Table 1. 
Table 1: Sets, Parameters and Decision variables of the OS approach

\begin{tabular}{|c|c|c|}
\hline Set name & Description & Alias \\
\hline$E$ & Elements in the original set & $\left\{e, e^{\prime}\right\}$ \\
\hline C & Elements to be selected & $c$ \\
\hline ET & Hours of an element & et \\
\hline M & Statistical measurements $\{$ Min, Mean, Max $\}$ & $m$ \\
\hline$S M$ & Subset of the statistical measurements $\{$ Min, Max $\}$ & $s m, S M \in M$ \\
\hline Parameter & Description & Unit \\
\hline $\operatorname{Res} E_{\text {et }, e}$ & Hourly residual loads of each element in original time series & {$[\mathrm{MW}]$} \\
\hline$R m p E_{e t, e}$ & Hourly ramping of loads of each element in original time series & {$[\mathrm{MW}]$} \\
\hline org_avg_var & Average element variance of original time series & {$[\mathrm{MW}]$} \\
\hline org_lvl $l_{m}$ & $\{$ Minimum, Mean, Maximum $\}$ load value of original time series & {$[\mathrm{MW}]$} \\
\hline org_rmp $p_{m}$ & $\{$ Minimum, Mean, Maximum\} ramp value of original time series & {$[\mathrm{MW}]$} \\
\hline VarEleme $_{e}$ & Variance of each element in original time series & \\
\hline CorElem $_{e, e^{\prime}}$ & Correlation between every combination of elements in original time series & \\
\hline Variable & Description & Domain \\
\hline$B \_a g g_{e}$ & Selected elements to be included in aggregated time series, 1 indicates inclusion of element $e$ & $\{0,1\}$ \\
\hline$B \_l v l_{s m, e, e t}$ & Auxiliary variable to select $\{$ Minimum, Maximum $\}$ load of aggregated time series, if 1 hour $e t$ in element $e$ is identified & $\{0,1\}$ \\
\hline$B_{\_} r m p_{s m, e, e t}$ & Auxiliary variable to select $\{$ Minimum, Maximum $\}$ ramp value of aggregated time series, if 1 hour $e t$ in element $e$ is identified & $\{0,1\}$ \\
\hline$B_{-} r m p_{s m, e, e t}$ & Auxiliary variable to select minimum ramp value of aggregated time series, if 1 hour $e t$ in element $e$ is identified & $\{0,1\}$ \\
\hline$D \_l v l_{m}$ & Difference in $\{$ minimum, mean, maximum $\}$ load value of aggregated and original time series & $\mathbb{R}_{+}$ \\
\hline$D_{-} r m p_{m}$ & Difference in $\{$ minimum, mean, maximum $\}$ ramp value of aggregated and original time series & $\mathbb{R}_{+}$ \\
\hline agg_var & Average element variance in aggregated time series & $\mathbb{R}$ \\
\hline$v a r_{-} d i f f$ & Difference in average element variance in aggregated and original time series & $\mathbb{R}$ \\
\hline $\operatorname{corPos}_{e, e^{\prime}}$ & Correlation between selected elements in aggregation & $\mathbb{R}$ \\
\hline$a g g \_l v l_{m}$ & $\{$ Minimum, Mean, Maximum $\}$ load value of aggregated time series & $\mathbb{R}$ \\
\hline$a g g_{-} r m p_{m}$ & $\{$ Minimum, Mean, Maximum $\}$ ramp value of aggregated time series & $\mathbb{R}$ \\
\hline B_matrix $e_{e, e^{\prime}}$ & Element combinations in selection & $\mathbb{R}$ \\
\hline res_agget,e & Hourly levels of selected elements & $\mathbb{R}$ \\
\hline ramp_agg et,e & Hourly ramping of selected elements & $\mathbb{R}$ \\
\hline
\end{tabular}

$\underset{B_{-} a g g_{e}}{\operatorname{Minimize}} \sum_{e, e^{\prime} \in E} \operatorname{corPos}_{e, e^{\prime}}+v a r_{-}$diff $+\sum_{m \in M} D_{-} l v l_{m}+D_{-} r m p_{m}$

Subject to

$$
\begin{aligned}
& \sum_{e \in E} B \_a g g_{e}=|C| \\
& \text { agg_var }=\sum_{e \in E}\left(B_{-} a g g_{e} \cdot \text { varElem }_{e}\right) /|C| \\
& v a r_{-} d i f f \geq a g g_{-} v a r-o r g \_a v g_{-} v a r \\
& v a r_{-} d i f f \geq o r g \_a v g_{-} v a r-a g g_{-} v a r \\
& B_{-} \text {matrix }_{e, e^{\prime}} \geq B_{-} a g g_{e}+B_{\_} a g g_{e^{\prime}} \quad \forall e, e^{\prime} \in E \\
& \operatorname{corPos}_{e, e^{\prime}} \geq \text { B_matrix }_{e, e^{\prime}} \cdot \text { CorElem }_{e, e^{\prime}} \quad \forall e, e^{\prime} \in E \\
& \operatorname{corPos}_{e, e^{\prime}} \geq-B_{-} \text {matrix }_{e, e^{\prime}} \cdot \text { CorElem }_{e, e^{\prime}} \quad \forall e, e^{\prime} \in E \\
& B \_l v l_{s m, e, e t} \leq B \_a g g_{e} \\
& B_{-} r m p_{s m, e, e t} \leq B \_a g g_{e} \\
& \sum_{e \in E} \sum_{e t \in E T} B_{-} l v l_{s m, e, e t}=1 \\
& \sum_{e \in E} \sum_{e t \in E T} B_{-} r m p_{s m, e, e t}=1 \\
& a g g_{-} l v l_{s m}=\sum_{e \in E} \sum_{e t \in E T} B \_l v l_{s m, e, e t} \cdot \operatorname{Res} E_{e t, e} \\
& a g g \_r m p_{s m}=\sum_{e \in E} \sum_{e t \in E T} B \_r m p_{s m, e, e t} \cdot R m p E_{e t, e} \\
& \text { res_agg } g_{\text {et } e}=B_{-} a g g_{e} \cdot \operatorname{Res} E_{\text {et }, e} \\
& a g g_{\_} l v l_{m}=\left(\sum_{e \in E} \sum_{e t \in E T} r e s_{-} a g g_{e t, e}\right) /(|E T| \cdot|C|) \\
& r a m p \_a g g_{e t, e}=B_{\_} a g g_{e} \cdot R m p E_{e t, e} \\
& a g g_{-} r m p_{m}=\left(\sum_{e \in E} \sum_{e t \in E T} r_{a m p \_a g g_{e t, e}}\right) /(|E T| \cdot|C|) \\
& D \_l v l \geq a g g_{-} l v l_{m}-o r g \_l v l_{m} \\
& D \_l v l \geq o r g_{-} l v l_{m}-a g g_{-} l v l_{m} \\
& \text { D_rmp } \geq a g g_{-} r m p_{m}-o r g \_r m p_{m} \\
& D \_r m p \geq o r g_{-} r m p_{m}-a g g_{-} r m p_{m} \\
& B \_a g g_{e}, B \_l v l_{s m, e, e t}, B \_r m p_{s m, e, e t} \in\{0,1\} \\
& \text { D_lvl } l_{m}, D \_r m p_{m} \geq 0 \\
& \forall s m \in S M, \forall e \in E, \forall e t \in E T \\
& \forall s m \in S M, \forall e \in E, \forall e t \in E T \\
& \forall s m \in S M \text {, } \\
& \forall s m \in S M \text {, } \\
& \forall s m \in S M \text {, } \\
& \forall s m \in S M \text {, } \\
& \forall e \in E, \forall e t \in E T \\
& \forall m \in\{\text { mean }\} \\
& \forall e \in E, \forall e t \in E T \\
& \forall m=\{\text { mean }\} \\
& \forall m \in M \text {, } \\
& \forall m \in M \text {, } \\
& \forall m \in M \text {, } \\
& \forall m \in M \text {, } \\
& \forall s m \in S M, \forall e \in E, \forall e t \in E T
\end{aligned}
$$


In the model (1)-(24), the main decision is which elements to include in the aggregated time selection, namely $B \_a g g_{e}$. All other decision variables are auxiliary variables, used to measure the penalty function which guides the selection of elements. The model complexity is strongly correlated to the number of elements being selected, which also makes it less suitable for selecting single hours. Equation (1) penalizes the difference in average, minimum and maximum level and ramping values between the aggregated and original time series, as well as the differences in average element variance. Furthermore, it minimizes the correlation between selected elements, which drives a selection of different elements. Equation (2) ensures that only a specific number of elements is chosen. In equation (3)-(5), the difference in average element variance is calculated, and correlation between elements in the selection is found in equation (6)-(8). Equations (9)-(10) ensure that elements targeting the minimum and maximum level and ramp values of the original time series are included in the selection. In equation (13)-(14) the corresponding level and ramp values of these selected elements are extracted. Equations (15)-(18), calculate average level and ramping values of the aggregated time series and equations (19)-(22) define the terms to be minimized, and by that ensure that the selection of elements has values close to the corresponding values of the original time series. Notice that, equation (6) adds an unwanted property, since not only the correlation between selected elements are punished, but actually also correlation between selected and non-selected elements. However the latter is punished with a lower weight. Defining the constraint like B_matrix $e_{e, e^{\prime}} \geq B_{-} a g g_{e} \wedge B_{-} a g g_{e^{\prime}}$, would fulfill the defined objective, but at the cost of the problem turning nonlinear. To avoid nonlinearities but still fulfilling our objective, constraint (6) could be defined as $B \_m a t r i x_{e, e^{\prime}} \geq B_{-} a g g_{e}+B_{-} a g g_{e^{\prime}}-1$, where $B \_$matrix $x_{e, e^{\prime}} \geq 0$. However, experimental results showed that this made the computational time explode, which essentially motivates the definition in (6), despite the slightly change in the definition of the objective.

\subsection{Selection criteria}

For all the strategies categorized as $G S \mathrm{~s}$, the explained aggregation approaches output groupings, and consequently, in order to achieve the aggregated time series, a selection from each group needs to be performed. As described in Section 2, many different selection criteria have been tried and discussed, and it is our aim to cover most of these. We note that a selection strategy also can consist of selecting multiple elements from each group, though in this work we only consider single selections. Table 2 contains the selection criteria analyzed and a definition according to each element type.

Table 2: Selection criteria for the $G S \mathrm{~s}$

\begin{tabular}{|c|c|c|}
\hline & \multicolumn{2}{|c|}{ Definition } \\
\hline Criteria & Hour & Day / Week \\
\hline Mean & $\begin{array}{l}\text { A constructed hour, being the average over all hours } \\
\text { in the group }\end{array}$ & $\begin{array}{l}\text { A constructed element where the } x^{t h} \text { hour is the av- } \\
\text { erage of the } x^{t h} \text { hour of all elements in the group. }\end{array}$ \\
\hline Minimum & The hour with minimum value in the group & $\begin{array}{l}\text { Summing all hours of each element, the element with } \\
\text { the minimum sum is selected. }\end{array}$ \\
\hline Maximum & The hour with maximum value in the group & $\begin{array}{l}\text { Summing all hours of each element, the element with } \\
\text { the maximum sum is selected. }\end{array}$ \\
\hline Random & A random hour of the group & A random element of the group \\
\hline cMean & The hour with a value closest to the group mean value & $\begin{array}{l}\text { For each element, the } x^{t h} \text { hour difference to the cor- } \\
\text { responding hour in the group mean element is calcu- } \\
\text { lated. Then summing all hourly differences of each el- } \\
\text { ement, and selecting the element with the minimum } \\
\text { sum of differences. }\end{array}$ \\
\hline
\end{tabular}




\subsection{Runtimes of the aggregation techniques}

The different aggregation strategies are summarized in Table 3. The HS and CA based aggregations are implemented in R-3.3.3 while the OPT based aggregations are implemented in GAMS 24.9.2 and solved using Cplex 12.7.1.0. For each strategy, the approximated time of performing an aggregated time series is listed. All solutions times are related to a laptop with an Intel(R) Core(TM)i5-6200U CPU with $2.30 \mathrm{GHz}$ and 8 GB RAM.

The optimized RLDC approximation appears to be the most time consuming approach with an hour spent on finding the aggregated time series. Due to the high complexity of this approach, the search is constrained by a time limit. Consequently, optimality is not ensured in the selected days. For most of the other techniques, aggregated instances are constructed in less than a second or in a couple of seconds, with the exceptions of the ES approach taking 3.5 minutes and the HS approach taking 35 sec (day selection). For the approaches applied to all element types, the times seem to be quite similar for each type with the exception of the SR approach being much more time consuming when days are selected. From our results there is no clear identification of one single element being consistently faster than the others but, it is seen that daily selections are more time consuming compared to weekly selections for all approaches.

Table 3: Time aggregation strategies analyzed in this paper. The time of the $G S$ category covers the time of forming groups and of performing all selections as described in Section 3.1

\begin{tabular}{|c|c|c|c|c|c|c|}
\hline \multirow[b]{2}{*}{ Approach } & \multirow[b]{2}{*}{ Category } & \multirow[b]{2}{*}{ Selection type } & \multirow[b]{2}{*}{ Supported Elements } & \multicolumn{3}{|c|}{ Approximated times (s) } \\
\hline & & & & Hours & Days & Weeks \\
\hline $\mathrm{SC}$ & $\mathrm{CA}$ & GS & Hours, Days, Weeks & $\sim 3$ & $\sim 2$ & $\sim 2$ \\
\hline $\mathrm{CC}$ & $\mathrm{CA}$ & GS & Hours, Days, Weeks & $\sim 3$ & $\sim 2$ & $\sim 2$ \\
\hline $\mathrm{LC}$ & $\mathrm{CA}$ & GS & Hours, Days, Weeks & $\sim 2$ & $\sim 3$ & $\sim 2.5$ \\
\hline DB & HS & GS & Hours, Days, Weeks & $\sim 2.7$ & $\sim 0.8$ & $\sim 0.35$ \\
\hline DX & HS & NGS & Hours, Days, Weeks & $\sim 0.2$ & $\sim 0.06$ & $\sim 0.06$ \\
\hline SR & HS & NGS & Hours, Days, Weeks & $\sim 4.5$ & $\sim 34.3$ & $\sim 8.1$ \\
\hline RL & HS & NGS & Hours, "Constructed-Days", "Constructed-Weeks" & $\sim 0.1$ & $\sim 0.1$ & $\sim 0.1$ \\
\hline ES & OPT & NGS & Weeks & - & - & $\sim 215$ \\
\hline $\mathrm{OA}$ & OPT & NGS & Days & - & $\sim 3600$ & - \\
\hline OS & OPT & NGS & Days, Weeks & - & $\sim 8$ & $\sim 3$ \\
\hline
\end{tabular}

\section{Test Cases}

\subsection{Data}

Investment technologies have been chosen such that VRE capacities, base-load capacity and flexible capacities are possible. Inspired by [6] and [17], we consider wind and PV as VRE, Nuclear and coal as base-load capacity and Open- and Combined-Cycle Gas Turbines (OCGT and CCGT) as the flexible capacities. The instances to be aggregated consist of hourly residual load profiles, calculated from hourly load profiles and hourly wind and PV availability profiles. In each model run only one profile of each type is used in the residual load calculation, corresponding to the system only containing one region. In order to enable aggregation performance analysis of years with low, high and middle overall wind availability, profiles representing the Danish energy load and supply conditions of the years 2014, 2015 and 2016 have been extracted directly from [8] (Table 4). The technological data of the remaining units are highly inspired by the values used in [5], but adjusted such that interesting investments, among different technologies, are made in the original solution. In this paper, the primary objective is to evaluate the ability of an aggregated 
model to capture the need for flexibility, and to provide optimal stability in a system heavily reliable on VREs. Consequently, it needs to be ensured that the original solution contains a high share of VRE, which is achieved by keeping the cost of the VRE technologies low. Focus has been on generating challenging instances that use several technologies, in order to make it easier to separate the wheat from the chaff in our study of aggregation techniques. Since we leave demands unchanged, any scaling of costs will not affect the aggregation techniques.

Table 4: Yearly supply and demands in MW. The supplies are found by assuming the maximum capacities to be the maximum production seen during the corresponding year.

\begin{tabular}{|c|c|c|c|c|c|}
\hline Year & \multicolumn{1}{c}{ Demand } & \multicolumn{2}{c}{ Wind } & \multicolumn{2}{c|}{ PV } \\
\multicolumn{2}{|c|}{ Yearly Sum } & \multicolumn{1}{c|}{ Max Cap } & \multicolumn{1}{c|}{ Yearly Sum } & \multicolumn{1}{c|}{ Max Cap } & \multicolumn{1}{c|}{ Yearly Sum } \\
\hline $\mathbf{2 0 1 4}$ & 33.355 .104 & 4886.6 & 13.028 .517 & 483 & 595.235 \\
$\mathbf{2 0 1 5}$ & 33.523 .940 & 5076.4 & 14.062 .862 & 496 & 604.149 \\
$\mathbf{2 0 1 6}$ & 33.799 .957 & 5245.3 & 12.627 .431 & 609 & 743.547 \\
\hline
\end{tabular}

\subsection{Model}

As discussed earlier, the increasing shares of VRE in the power generation mix, turns the focus on the insurance of reliability, from not just being achieved, but being achieved through an optimal capacity expansion mix, resulting in the least costs [25]. To achieve this, short-term temporal load variations are included in the capacity expansion model, resulting in a 'two stage' model. The first stage decides on the optimal building decisions while optimal operational decisions of the installed capacities are made in the second stage. The objective of such a model is to find the optimal balance between investment and production costs. In other words, expansion costs are minimized subject to technical and operational constraints [9]. A model like this has several applications, some of them being to determine the optimal electricity generation capacity mix at a given future time, determine the future investments needed to supply a growing electricity demand at minimum cost, analyzing effects of the different constraints on the system costs and analyzing the value of flexibility options by comparing the total system cost with and without each option [5]. In this paper the focus is on the analysis of flexibility options to provide stability in a system heavily reliant on VREs. The chosen model structure is highly inspired by the one used in [5], but for simplicity we disregard reserves in the model which normally are used to account for the uncertainty in the predictions of the load and availability profiles. Also, as opposed to [5], we include VRE capacities as variables instead of parameters, to enable the analysis of aggregation affecting VRE capacity investments. In the following a mathematical formulation of the model is seen. Sets, parameters and variables are listed in Table 5. From this, it is seen that shut-downs $\left(v_{i j}\right)$ are defined as continuous variables. Originally, these are binary decisions, but since they only appear in constraint (30), where all variables are binary, any optimal solution will not contain fractional values. 
Table 5: Sets, Parameters and Variables of the CEUCM

\begin{tabular}{|c|c|c|}
\hline Set name & Description & Alias \\
\hline $\begin{array}{c}J \\
I \\
I^{W} \\
I^{S} \\
I^{T}\end{array}$ & $\begin{array}{l}\text { Time periods of the analyzed time series } \\
\text { Units, which potentially are built } \\
\text { Units of wind type } \\
\text { Units of solar type } \\
\text { Units of thermal type (Nuclear, coal, CCGTs and OCGTs) }\end{array}$ & $\begin{array}{l}\left\{j, j^{\prime}\right\} \\
\quad i \\
I^{W} \subset I \\
I^{S} \subset I \\
I^{T} \subset I\end{array}$ \\
\hline Variable & Description & Domain \\
\hline $\begin{array}{l}y_{i} \\
u_{i j} \\
z_{i j} \\
v_{i j} \\
x_{i j} \\
w_{i j}\end{array}$ & $\begin{array}{l}\text { Building decision for unit } i \text {, if } 1 \text { the unit is built } \\
\text { Commitment state, if } 1 \text { unit } i \text { is online in time } j \\
\text { Start-up decision, if } 1 \text { unit } i \text { is started up in time } j \\
\text { Shut-down decision, if } 1 \text { unit } i \text { is shut down in time } j \\
\text { Power output decision, amount of energy generated by unit } i \text { in time } j \\
\text { Power generation over minimum capacity of unit } i \text { in time } j\end{array}$ & $\begin{aligned} & \{0,1\} \\
& \{0,1\} \\
& \{0,1\} \\
\mathbb{R}_{+} & (\text {or }\{0,1\}) \\
& \mathbb{R}_{+} \\
& \mathbb{R}_{+}\end{aligned}$ \\
\hline
\end{tabular}

Minimize $\sum_{i \in I} C_{i}^{F O M} y_{i}+\sum_{i \in I} \sum_{j \in J} C_{i j}^{V O M} x_{i j}+C_{i}^{S T U P} z_{i j}$

Subject to

$$
\begin{gathered}
\sum_{i \in I} x_{i j} \geq D_{j} \\
x_{i j} \leq \bar{P}_{i} y_{i} \\
x_{i j} \leq \bar{P}_{i} C F_{j}^{W I N D} \\
x_{i j} \leq \bar{P}_{i} C F_{j}^{S O L A R} \\
u_{i j}-u_{i j-1}=z_{i j}-v_{i, j} \\
w_{i j}=x_{i j}-u_{i j} \underline{P}_{i} \\
w_{i j} \leq u_{i j}\left(\bar{P}_{i}-\underline{P}_{i}\right) \\
w_{i j}-w_{i j-1} \leq \bar{R}_{i}^{U} \\
w_{i j-1}-w_{i j} \leq \bar{R}_{i}^{D} \\
u_{i j} \geq \sum_{j^{\prime}>j-\bar{M}_{i}^{U}}^{z_{i j^{\prime}}} \\
1-u_{i j} \geq \sum_{j^{\prime}>j-\bar{M}_{i}^{D}}^{j} v_{i j^{\prime}} \\
y_{i}, u_{i j}, v_{i j}, z_{i j} \in\{0,1\} \\
x_{i j}, w_{i j} \geq 0
\end{gathered}
$$$$
\forall j \in J
$$$$
\forall i \in I, \forall j \in J
$$$$
\forall i \in I^{W}, \forall j \in J
$$$$
\forall i \in I^{S}, \forall j \in J
$$$$
\forall i \in I^{T}, \forall j \in J \backslash\{1\}
$$$$
\forall i \in I^{T}, \forall j \in J
$$$$
\forall i \in I^{T}, \forall j \in J
$$$$
\forall i \in I^{T}, \forall j \in J \backslash\{1\}
$$$$
\forall i \in I^{T}, \forall j \in J \backslash\{1\}
$$$$
\forall i \in I^{T}, \forall j \in J
$$$$
\forall i \in I^{T}, \forall j \in J
$$$$
\forall i \in I, \forall j \in J
$$$$
\forall i \in I, \forall j \in J
$$ 
The objective function (25) minimizes fixed and variable costs of investments and operations. The fixed costs $C_{i}^{F O M}$ cover investment costs and fixed O\&M costs, while the variable costs $C_{i j}^{V O M}$ consist of fuel costs, variable O\&M costs and variable operational costs. Constraint (26) ensures energy balance while constraints (27)-(29) handle the capacities. From these it is seen that curtailment is allowed at no extra cost. Constraints (30)-(36) represent the unit commitment, meaning that these account for the commitment state, updating of shut-down and start-ups, ramping restrictions and minimum up- and down times. Constraint (31) defines the auxiliary variable $w_{i j}$ as the power generated above the minimum level of the unit. In (37)-(38) the domain of the variables are defined. Note, that constraints (27) and (31) implicitly secure zero commitment state for non-built units.

\subsection{Computational difficulty of the proposed CEUCM}

The proposed CEUCM is implemented in GAMS 24.9.2 and solved using Cplex 12.7.1.0 with standard settings for MIP. It is solved on a server with a node configuration of 2x Intel Xeon Processor 2660v3 (10 cores, $2.60 \mathrm{GHz}$ ) and $128 \mathrm{~GB}$ RAM. We solve the original problem to a $0.15 \%$ gap and then we fix the investments and solve the remaining problem to optimality. We are aware that there is a risk of getting sub-optimal investments, but we believe that the $0.15 \%$ gap is below the uncertainty of the instances.

Solving the CEUCM for each of the 3 years results in the solution specifics listed in Table 24 in Appendix C. It is seen that the optimal investment strategy includes investment of all technology types, which indeed makes it a thorough solution. Furthermore, optimal investments turn out to be the exact same for all the 3 years, indicating consistency in the 3 data sets. Regarding total system costs, satisfying demand in year 2015 is the overall cheapest, while 2016 is the most expensive year. Looking at Table 6, this seems to correlate with the solution time of the model, since the model of 2015 takes the longest time while the model of 2016 is solved the fastest. These results indicate that the computational difficulty of the problem is higher when more wind is available, but also that too low wind availability can make it harder to solve. In the following sections these original solutions will be referred to as Benchmark Solutions (BS).

Table 6: Solution times of the CEUCM for the 3 years

\begin{tabular}{|c|c|c|c|}
\hline Model type & $\mathbf{2 0 1 4}$ & $\mathbf{2 0 1 5}$ & $\mathbf{2 0 1 6}$ \\
\hline CEUCM & $4787.84 \mathrm{~s}$ & $5748.98 \mathrm{~s}$ & $4304.38 \mathrm{~s}$ \\
\hline
\end{tabular}

\section{Validation}

A core point in time aggregation is the validation of the aggregated data, since this addresses the errors committed by using aggregated instances instead of original instances. As discussed in Section 2, systematic validation is seldom seen in the literature, and whenever it is performed, it is typically limited to, what we refer to as data validation. This appears to be a good guiding criterion, though for a real validation it should be combined with a model validation. In the following, we give a detailed description of the two validation approaches.

\subsection{Data Validation}

Data validation measures the degree to which the aggregated time series replicates the original time series. Typically this is addressed by looking at the difference between the RLDCs of the original and the aggre- 
gated data. In order to measure the difference between the RLDCs a distance metric has to be chosen. With the goal of evaluating the aggregations on their ability to capture the extreme points, a NRMSE is often seen as the performance measure when the two RLDCs are compared (see e.g. $[6,17,28]$ ). This error metric punishes large differences and by that focuses on the single large differences rather than the amount of points that differ. When deciding on optimal investments the extreme points are believed to be crucial, which makes the NRMSE a suitable distance metric. The definition is given as follows

$$
\mathrm{NRMSE}=\frac{\sqrt{\sum_{t \in T}\left(R L D C_{t}-\overline{R L D C_{t}}\right)^{2}}}{|T|},
$$

where $R L D C_{t}$ is the original RLDC, $\overline{R L D C}_{t}$ is the approximated RLDC and $|T|$ is the amount of hours in the original instance. The data validation procedure is summarized in Procedure 1.

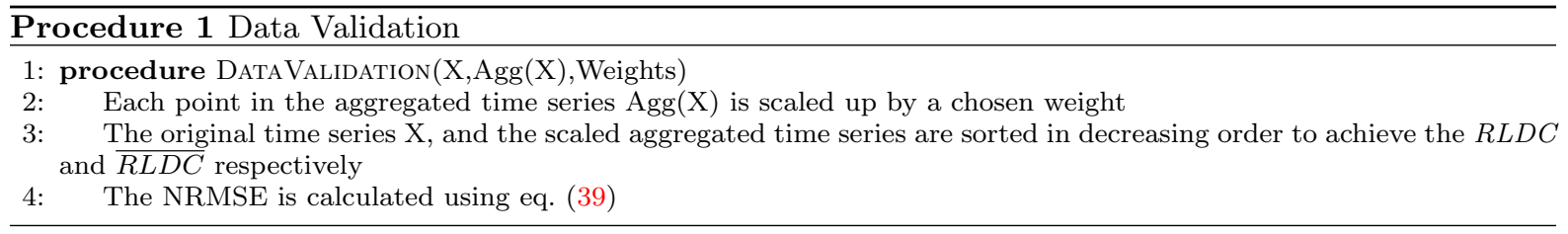

One of the disadvantages of using duration curves rather than chronological ordered curves is that, in general, the different levels of power generation can be analyzed, but no assesment of power generation at or between different times can be made. Due to time chronology being lost in the DC's time-scale, relationships are lost [25]. Consequently this validation provides no information of the ability to satisfy inter-temporal constraints like ramp-rates, start-ups, and minimum up and down times. Contrary, as discussed in Section 2 , the known challenges of aggregation is underestimation of flexible and base load capacities and validation according to RLDC replication then makes good sense since this reveals the need for these capacities (See Figure 2).

\subsection{Model Validation}

Model validation measures the ability of the aggregated problem to replicate the solution achieved from the original problem having higher computational difficulty. Essentially, the solution of the aggregated problem is compared to the solution achieved from solving the original problem. Our main evaluation criteria focus on the optimal investment decisions and the solution times. Furthermore, we also compare the maximum used capacities, the generation mix, the number of shut-down/start-up cycles and the overall system cost. To compare the latter, we need to scale up the aggregated solution. This is done by solving the aggregated problem to obtain optimal investment decisions, and then fix these when solving the original problem. This solution will then show the feasibility of the aggregated solution, especially regarding ramping and demand satisfaction. Furthermore it reveals the total system costs, the used capacities and the generation mix based on one year of hourly resolution. The model validation procedure is outlined in Procedure 2.

As discussed earlier, the challenge of the model validation approach is that aggregation often is used as a necessity due to intractability of the original problem. Consequently, a solution to the original problem cannot be obtained, making model validation impossible. In this work, we combine a model with data instances such that tractability is ensured and then we assume that the found results will be valid for larger problems as well. 


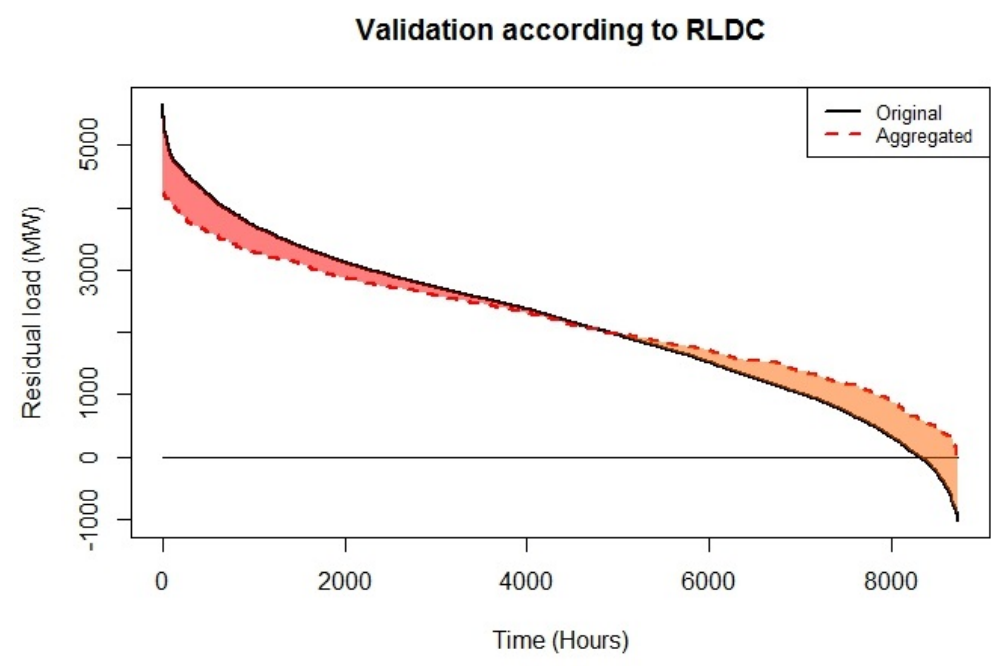

Fig. 2: Data validation of aggregation using RLDC. In the above graph, the peak residuals are underestimated, resulting in an underestimation of flexible capacities (red area). The last part of the RLDC is overestimated, resulting in an overstimation of base-load capacities (orange area).

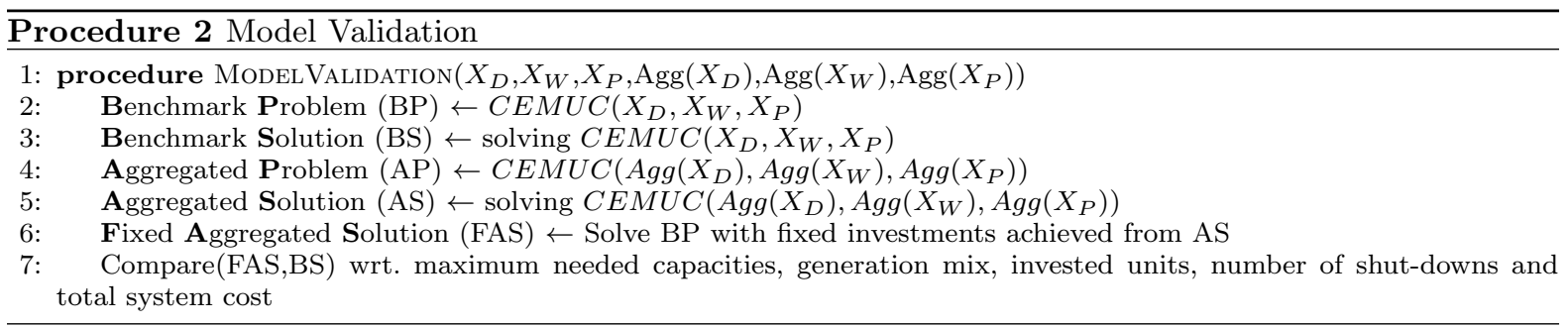

\section{Model Validation of the Aggregation Strategies}

Since the core of aggregation is to find good quality solutions in less time, the proposed model validation is, in our opinion, the best way of validating aggregated time series. Model validation, is exactly providing the basis for balancing solution time against solution quality. Hence, we structure this section by first validating the aggregated time series according to solution quality, and then afterwards look into the data validation measures and compare these to the results of the model validation. Defining a single aggregation to be dependent both on year, element type and selection criteria, we end up having 219 single aggregations in our analysis, of which 180 represent the GSs and 39 represent the $N G S$ s.

\subsection{General Performance of Aggregating}

By following Procedure 2 for all the single aggregations, we found that the resulting solutions can be grouped into 9 different categories according to their investments. In the following we refer to these as Investment Selections (IS) 1-9, see Table 7. Before studying which of the 219 single aggregations finds 
which of the 9 ISs, we provide an analysis of the ISs. Afterwards, the coupling between single aggregations and ISs enable an analysis of the aggregation performances.

\subsubsection{Performance Index (PI) Definition}

As Procedure 2 suggests, the solutions are evaluated according to their performance within 5 categories $(\Lambda)$, namely, maximum capacities, generation mix, invested units, number of shut-downs and overall system costs. We define a Performance Index (PI) based on differences $(D)$ between the aggregated and original solution within the mentioned categories. For each of the ISs, the difference to the original solution in each category is calculated both according to over- and underestimation $\left(\overline{D_{\lambda}}, \underline{D_{\lambda}}\right)$. To ensure equal importance of the 5 categories, differences within each category are normalized. The PI of the IS is then defined as the sum of the differences for all categories and for over- and underestimation. Since the differences for unit capacity and generation mix are split out on different units, these are summed and the resulting value is the one used to calculate the PI. Consequently, the proposed PI does not distinguish between which types of capacities that are not captured correctly, but punishes all equally. Using the proposed notation, the PI of year $y$ is mathematical defined as,

$$
\mathrm{PI}^{y}=\sum_{\lambda \in \Lambda}\left(\frac{\overline{D_{\lambda}^{y}}}{\left\|\overline{D_{\lambda}^{y}}\right\|}+\frac{D_{\lambda}^{y}}{\| \underline{D_{\lambda}^{y} \|}}\right)
$$

Due to the normalization, very small differences can have a fairly high impact. In order to make the PI robust to noise we introduce a tolerance $\epsilon$ used to ensure that differences below $10^{-4}$ are ignored,

$$
D= \begin{cases}D & \text { if } D>\epsilon \\ 0 & \text { otherwise }\end{cases}
$$

Furthermore, aggregating these problem instances may lead to infeasibilities due to ramping restrictions or unsatisfied demand. Since this is undesirable, infeasibilities are introduced as a performance category of its own, ensuring the PI to reflect the punishment of infeasibilities. The more infeasible an aggregated problem instance is, the more it is punished.

\subsubsection{Model Performance of the ISs}

Despite the outcome of all the single aggregations resulted in only 9 different investment strategies, these potentially can appear for any of the 3 years, and hence up to 27 different solutions could be seen. Consequently, each IS is associated with a yearly PI. However, not all ISs appear for all 3 years, essentially the strategies of 2016 cover 5 of the 9 ISs, investment strategies of 2014 cover 6 and for 2015 all 9 ISs are seen. In the end, 20 different solutions are seen and by that 20 different PIs spread over the 3 years. The values of the 20 different solutions are listed in Table 7 and Table 24 in Appendix C, where also the solution values of the BSs for the corresponding year are reported. The differences between the BSs and the ISs, in each of the 5 performance categories, are listed in Table 8. Differences for all units are summed both according to over- and underestimation.

A first thing to notice is, that each of the ISs appearing for multiple years seem to have similar differences for the different years. Furthermore, it seems that each IS, and by that each aggregation strategy, tends to under- and overestimate the generation mix equally while both according to capacities, amount of units built and amount of shutdowns the majority of the differences lie in the underestimation area. Consequently, the investment strategies arising from the aggregations select too few units, which potentially can be a reason for the fewer start-up/shutdown cycles. Further reasons could be the potential inability of the aggregations to capture an optimal commitment plan for the units. The very similar amount of over- and underestimation 
Table 7: Investments and objective values of the 9 different IS achieved among the 219 single aggregations. For investments, the numbers indicate the amount of units invested in, and all units within each category are identical. The objective value is provided for each of the 3 years. Objective denotes investment and operational costs, hence smaller values are better.

\begin{tabular}{|c|c|c|c|c|c|c|c|c|c|}
\hline \multirow[t]{2}{*}{ IS } & \multicolumn{6}{|c|}{ Investments } & \multicolumn{3}{|c|}{ Objective Values } \\
\hline & Wind & PV & Nuclear & Coal & CCGT & OCGT & 2014 & 2015 & 2016 \\
\hline BS & 6 & 6 & 1 & 3 & 1 & 1 & 695898021 & 688163336 & 777128914 \\
\hline IS 1 & 6 & 6 & $\overline{1}$ & 3 & - & $\overline{1}$ & 695995726 & 688435622 & 777281868 \\
\hline IS 2 & 6 & 6 & 1 & 2 & - & 1 & 699189926 & 692670977 & 782443912 \\
\hline IS 3 & 6 & 6 & 1 & 3 & - & - & 699537701 & 691650977 & - \\
\hline IS 4 & 6 & 6 & - & 3 & - & 1 & 698519665 & 691147531 & 779665388 \\
\hline IS 5 & 6 & 6 & - & 4 & - & 1 & 698533890 & 691124557 & 779690518 \\
\hline IS 6 & 6 & 6 & - & 3 & 1 & 1 & - & 690026363 & - \\
\hline IS 7 & 6 & 6 & - & 4 & - & - & - & 696038908 & - \\
\hline IS 8 & 6 & 6 & 1 & 4 & - & 1 & 696032672 & 688473986 & 777307169 \\
\hline IS 9 & 6 & 6 & 2 & 4 & - & 1 & - & 688595948 & - \\
\hline
\end{tabular}

Table 8: Percentage deviation of the 9 ISs from the original solution within each performance category. Due to none of our aggregated problem instances being infeasible, the infeasibility category is ignored.

\begin{tabular}{|c|c|c|c|c|c|c|c|c|c|c|c|c|c|c|c|}
\hline \multirow[b]{2}{*}{ IS } & \multicolumn{3}{|c|}{ Capacity } & \multicolumn{3}{|c|}{ Generation Mix } & \multicolumn{3}{|c|}{ \# Units } & \multicolumn{3}{|c|}{ \# Shut-Downs } & \multicolumn{3}{|c|}{ System Costs } \\
\hline & 2014 & 2015 & 2016 & 2014 & 2015 & 2016 & 2014 & 2015 & 2016 & 2014 & 2015 & 2016 & 2014 & 2015 & 2016 \\
\hline \multicolumn{16}{|c|}{ Underestimation } \\
\hline IS 1 & 2.99 & 2.76 & 2.65 & 0.09 & 0.11 & 0.10 & 5.56 & 5.56 & 5.56 & 1.81 & 2.89 & 2.44 & 0 & 0 & 0 \\
\hline IS 2 & 13.57 & 13.83 & 13.47 & 1.36 & 1.75 & 2.16 & 11.11 & 11.11 & 11.11 & 0.11 & 1.40 & 0 & 0 & 0 & 0 \\
\hline IS 3 & 4.99 & 4.19 & - & 0.19 & 0.21 & - & 11.11 & 11.11 & - & 16.06 & 18.15 & - & 0 & 0 & - \\
\hline IS 4 & 10.40 & 10.42 & 10.38 & 1.27 & 1.41 & 1.25 & 11.11 & 11.11 & 11.11 & 17.87 & 17.25 & 17.39 & 0 & 0 & 0 \\
\hline IS 5 & 10.40 & 10.42 & 10.38 & 1.27 & 1.41 & 1.25 & 11.11 & 11.11 & 11.11 & 17.77 & 17.25 & 17.39 & 0 & 0 & 0 \\
\hline IS 6 & - & 7.66 & - & - & 1.30 & - & - & 5.56 & - & - & 10.87 & - & - & 0 & - \\
\hline IS 7 & - & 11.85 & - & - & 1.51 & - & - & 16.67 & - & - & 43.67 & - & - & 0 & - \\
\hline IS 8 & 2.99 & 2.76 & 2.65 & 0.09 & 0.11 & 0.10 & 5.56 & 5.56 & 5.56 & 1.91 & 2.89 & 2.44 & 0 & 0 & 0 \\
\hline IS 9 & - & 2.76 & - & - & 0.11 & - & - & 5.56 & - & - & 2.29 & - & - & 0 & - \\
\hline \multicolumn{16}{|c|}{ Overestimation } \\
\hline IS 1 & 0 & 1.04 & 0.42 & 0.17 & 0.21 & 0.18 & 0 & 0 & 0 & 0 & 0 & 0 & 0.01 & 0.04 & 0.02 \\
\hline IS 2 & 3.62 & 0.75 & 3.88 & 1.43 & 1.86 & 2.24 & 0 & 0 & 0 & 0 & 0 & 0.42 & 0.47 & 0.66 & 0.68 \\
\hline IS 3 & 0.81 & 1.042 & - & 0.71 & 0.73 & - & 0 & 0 & - & 0 & 0 & - & 0.52 & 0.51 & - \\
\hline IS 4 & 0.44 & 0.56 & 0.79 & 1.64 & 1.81 & 1.61 & 0 & 0 & 0 & 0 & 0 & 0 & 0.38 & 0.43 & 0.36 \\
\hline IS 5 & 1.34 & 2.16 & 1.67 & 1.64 & 1.81 & 1.61 & 5.56 & 5.56 & 5.56 & 0 & 0 & 0 & 0.38 & 0.43 & 0.33 \\
\hline IS 6 & - & 0.16 & - & - & 1.45 & - & - & 0 & - & - & 0 & - & - & 0.27 & - \\
\hline IS 7 & - & 1.74 & - & - & 2.59 & - & - & 5.56 & - & - & 0 & - & - & 1.14 & - \\
\hline IS 8 & 1.34 & 2.78 & 1.67 & 0.16 & 0.21 & 0.17 & 5.56 & 5.56 & 5.56 & 0 & 0 & 0 & 0.02 & 0.05 & 0.02 \\
\hline IS 9 & - & 2.78 & - & - & 0.21 & - & - & 11.11 & - & - & 0 & - & - & 0.06 & - \\
\hline
\end{tabular}

of generation mix is caused by the aggregated solutions 'just' shifting around generation on different units. Hence, the ISs still hold a similar overall generation level to the BS, however, the level is slightly higher in the aggregated solutions. A higher level of overall generation is an indicator of insufficient capture of rampings. If the rampings are poorly described in the aggregation, investments will not be efficient in terms of flexibility. To satisfy demand and to respect rampings, the optimal solution will increase its generation in specific hours to maximize the possible production in the next hour. For the aggregated problem, capturing these rampings can be a challenge leading to the necessity of producing power on more inefficient and 
expensive units to satisfy the demand. A consequence of this is also the overestimation of total system costs which is seen for every IS.

All ISs capture the VRE units correctly. The challenge is to capture the flexible units and by that also the base-load capacity units. Each of the BSs invests in one unit of OCGT and one of CCGT and even though the overall production on the two units are very similar, installed capacity is twice as large for the CCGT compared to the OCGT. Nevertheless, most of the ISs invest in the OCGT unit, and only IS 6 invests in the CCGT unit. However, IS 6 overestimates the production on the gas units which causes a lack of nuclear investment. With the flexible units being more expensive in production compared to producing on nuclear based units, IS 6 tends to overestimate total system cost, leading to an overall average performance, dispite the correct caption of gas units.

Contrarily, with only a single CCGT unit differentiating the investments of IS 1 from those of the BS, IS 1 closely replicates the BS, with only small deviations, causing IS 1 to achieve the best PI for each year, as seen in Table 9. IS 8 is also performing well, but apart from the missing CCGT investment, IS 8 also overinvests in coal units, which gives it a second best ranking within each year. Regarding worst performing strategies, the PI in Table 9 points at ISs 2, 5 and 7. Common for IS 5 and 7 is that they invest too much in coal units, produce too much on these and underinvest in other thermal units. Contrary, IS 2 actually underinvest in coal and account for the missing capacity by increasing production on nuclear units. From an investment point of view, IS 2 only deviates by a single coal unit investment from IS 1 , which makes the big performance difference a bit surprising.

Table 9: PI of the ISs for the 3 years. PI is calculated as described in Section 6.1.1 and smaller values indicate better results.

\begin{tabular}{|c|c|c|c|}
\hline IS & 2014 & 2015 & 2016 \\
\hline IS 1 & 0.1 & 0.28 & 0.15 \\
\hline IS 2 & 5.76 & 4.75 & 7 \\
\hline IS 3 & 3.76 & 1.98 & - \\
\hline IS 4 & 5.47 & 3.50 & 4.54 \\
\hline IS 5 & 6.71 & 4.44 & 5.79 \\
\hline IS 6 & - & 2.12 & - \\
\hline IS 7 & - & 6.61 & - \\
\hline IS 8 & 1.48 & 1.27 & 1.51 \\
\hline IS 9 & - & 1.77 & - \\
\hline
\end{tabular}

\section{Concluding on the IS Performance}

Overestimation of total system cost and generation mix, and underestimation of investments, especially regarding the flexible unit capacity, are generally seen for all aggregation techniques, which also is observed in the literature $([1,32,27])$. An interesting observation is that the benchmark investment strategy is not found by any of the 219 single aggregations and this illustrates that aggregation is a simplification technique where solution time and quality need to be balanced. Though, it is also important to notice that in terms of overall system costs, the best performing IS only deviates by $\sim \$ 100.000$ from the BS, corresponding to $0.01 \%$ of the BS objective value. With a solution time decrease of up to $98 \%$ (showed later) this is a trade-off that many would make and this clearly illustrates why aggregation techniques are so popular. In the light of an optimization model being a simplification of the real world problem, a deviation of $0.01 \%$ in overall system cost still provides a very good solution. For the majority of the ISs seen, the overestimation of total system costs is less than $1 \%$ although for some, up to $3 \%$ is seen. Though these deviations might increase as the problem size increases. 
In the following we study the performance of the single aggregations in order to compare their performance and complexity level.

\subsection{Comparing Performances of the Single Aggregations}

A performance overview of the 180 single aggregations within the $G S$ category can be seen in Table 10 while Table 11 shows the 39 NGS categorized single aggregations. A first thing to notice is that the average performances, over all years and all element types, in general, are better for the NGSs compared to the performances of the GSs. The main reason is, that the performance of the GSs are very dependent on the selection criteria used. In general, the GSs performances is dominated by some selection criteria performing significantly worse than others. Furthermore the performance of the single aggregations seems to be highly dependent on the type of element selected. Especially for the $G S$ s we see a clear division between the performance of aggregations selecting hours and those selecting either days or weeks. Consequently, we initiate the comparison of the aggregation techniques, by looking at the performance according to element type and selection criteria.

Table 10: Found ISs of the GSs

\begin{tabular}{|c|c|c|c|c|c|c|c|c|c|c|c|c|c|}
\hline \multirow[b]{2}{*}{ Strategy } & \multicolumn{4}{|c|}{ Hour } & \multicolumn{4}{|c|}{ Day } & \multicolumn{4}{|c|}{ Week } & \multirow[b]{2}{*}{ AVG PI } \\
\hline & 2014 & 2015 & 2016 & AVG & 2014 & 2015 & 2016 & AVG & 2014 & 2015 & 2016 & AVG & \\
\hline DB_Mean & IS 5 & IS 5 & IS 5 & 5.65 & IS 1 & IS 1 & IS 1 & 0.14 & IS 2 & IS 2 & IS 1 & 3.55 & 3.11 \\
\hline DB_cMean & IS 5 & IS 5 & IS 5 & 5.65 & IS 1 & IS 6 & IS 4 & 2.22 & IS 1 & IS 2 & IS 1 & 1.63 & 3.17 \\
\hline DB_Min & IS 4 & IS 5 & IS 5 & 5.23 & IS 2 & IS 1 & IS 1 & 2.06 & IS 2 & IS 2 & IS 1 & 3.55 & 3.62 \\
\hline DB_Max & IS 5 & IS 5 & IS 5 & 5.65 & IS 4 & IS 7 & IS 4 & 5.54 & IS 3 & IS 3 & IS 4 & 3.42 & 4.87 \\
\hline DB_Random & IS 5 & IS 5 & IS 5 & 5.65 & IS 1 & IS 1 & IS 1 & 0.14 & IS 4 & IS 4 & IS 1 & 3.04 & 2.94 \\
\hline DB - PI(AVG) & 6.47 & 4.44 & 5.79 & 5.57 & 2.25 & 1.92 & 1.90 & 2.02 & 4.15 & 3.94 & 1.02 & 3.04 & 3.54 \\
\hline SC_Mean & IS 5 & IS 5 & IS 5 & 5.65 & IS 1 & IS 1 & IS 4 & 1.61 & IS 2 & IS 2 & IS 2 & 5.34 & 4.36 \\
\hline SC_cMean & IS 8 & IS 8 & IS 8 & 1.42 & IS 1 & IS 1 & IS 1 & 0.14 & IS 2 & IS 2 & IS 2 & 5.84 & 2.47 \\
\hline SC_Min & IS 5 & IS 8 & IS 8 & 3.16 & IS 1 & IS 1 & IS 1 & 0.14 & IS 1 & IS 2 & IS 2 & 3.92 & 2.41 \\
\hline SC_Max & IS 8 & IS 5 & IS 8 & 2.48 & IS 4 & IS 1 & IS 1 & 1.97 & IS 4 & IS 3 & IS 1 & 2.53 & 2.32 \\
\hline SC_Random & IS 5 & IS 8 & IS 8 & 3.16 & IS 1 & IS 1 & IS 1 & 0.14 & IS 1 & IS 1 & IS 1 & 0.14 & 1.15 \\
\hline SC - PI(AVG) & 4.62 & 2.54 & 2.36 & 3.18 & 1.09 & 0.28 & 1.02 & 0.80 & 3.40 & 3.94 & 4.26 & 3.65 & 2.54 \\
\hline CC_Mean & IS 5 & IS 5 & IS 8 & 4.22 & IS 1 & IS 1 & IS 1 & 0.14 & IS 1 & IS 2 & IS 2 & 3.92 & 2.76 \\
\hline CC_cMean & IS 5 & IS 5 & IS 5 & 5.65 & IS 1 & IS 6 & IS 1 & 0.76 & IS 1 & IS 2 & IS 2 & 3.92 & 3.44 \\
\hline CC_Min & IS 5 & IS 5 & IS 5 & 5.65 & IS 1 & IS 1 & IS 1 & 0.14 & IS 1 & IS 1 & IS 2 & 2.43 & 2.74 \\
\hline CC_Max & IS 5 & IS 5 & IS 5 & 5.65 & IS 1 & IS 1 & IS 1 & 0.14 & IS 4 & IS 1 & IS 1 & 1.97 & 2.59 \\
\hline CC_Random & IS 5 & IS 5 & IS 5 & 5.65 & IS 1 & IS 1 & IS 1 & 0.14 & IS 1 & IS 1 & IS 4 & 1.61 & 2.47 \\
\hline CC - PI(AVG) & 6.71 & 4.44 & 4.94 & 5.36 & 0 & 0.65 & 0.15 & 0.27 & 1.09 & 2.07 & 5.14 & 2.77 & 2.80 \\
\hline LC_Mean & IS 8 & IS 9 & IS 5 & 3.02 & IS 1 & IS 1 & IS 1 & 0.14 & IS 2 & IS 2 & IS 2 & 5.84 & 3.00 \\
\hline LC_cMean & IS 8 & IS 8 & IS 8 & 1.42 & IS 1 & IS 1 & IS 4 & 1.61 & IS 4 & IS 2 & IS 2 & 5.74 & 2.92 \\
\hline LC_Min & IS 5 & IS 8 & IS 8 & 3.16 & IS 4 & IS 6 & IS 1 & 2.58 & IS 2 & IS 6 & IS 2 & 4.96 & 3.57 \\
\hline LC_Max & IS 8 & IS 8 & IS 8 & 1.42 & IS 1 & IS 1 & IS 4 & 1.61 & IS 4 & IS 1 & IS 1 & 1.97 & 1.67 \\
\hline LC_Random & IS 8 & IS 8 & IS 8 & 1.42 & IS 1 & IS 1 & IS 1 & 0.14 & IS 1 & IS 1 & IS 1 & 0.14 & 0.57 \\
\hline LC - PI(AVG) & 2.53 & 1.37 & 2.36 & 2.09 & 1.09 & 0.65 & 1.90 & 1.22 & 4.49 & 2.44 & 4.26 & 3.73 & 2.34 \\
\hline
\end{tabular}

\subsubsection{Impact of Element Types on the Aggregation Performance}

Table 12 gives an overview of the different ISs seen for each element type. When selecting hours, IS 5 dominates the outcomes by appearing in $60 \%$ of the $G S$ s and $55.6 \%$ of the $N G S$ s. Consequently, both for 
Table 11: Found ISs of the NGSs

\begin{tabular}{|c|c|c|c|c|c|c|c|c|c|c|c|c|c|}
\hline \multicolumn{6}{|c|}{ Hour } & \multicolumn{3}{|c|}{ Day } & \multicolumn{4}{|c|}{ Week } & \multirow[b]{2}{*}{ AVG PI } \\
\hline Strategy & 2014 & 2015 & 2016 & AVG & 2014 & 2015 & 2016 & AVG & 2014 & 2015 & 2016 & AVG & \\
\hline DX & IS 5 & IS 5 & IS 5 & 5.65 & IS 1 & IS 1 & IS 1 & 0.14 & IS 1 & IS 1 & IS 1 & 0.14 & 1.98 \\
\hline SR & IS 1 & IS 1 & IS 1 & 0.14 & IS 1 & IS 1 & IS 4 & 1.61 & IS 1 & IS 1 & IS 1 & 0.14 & 0.63 \\
\hline RL & IS 8 & IS 5 & IS 5 & 3.91 & IS 5 & IS 5 & IS 5 & 5.65 & IS 5 & IS 5 & IS 5 & 5.65 & 5.07 \\
\hline ES & - & - & - & - & - & - & - & - & IS 1 & IS 1 & IS 4 & 1.61 & 1.61 \\
\hline $\mathrm{OA}$ & - & - & - & - & IS 1 & IS 1 & IS 4 & 1.61 & - & - & - & - & 1.61 \\
\hline OS & - & - & - & - & IS 1 & IS 5 & IS 1 & 1.53 & IS 1 & IS 1 & IS 1 & 0.14 & 0.84 \\
\hline
\end{tabular}

GSs and NGSs, hourly selections show the worst performance according to the average PI. On the contrary, the best chances of good performance is seen when selecting days in a $G S$ or weeks in an NGS, since IS 1 appears in $78.1 \%$ of the $G S$ outcomes and $73 \%$ of the $N G S$ outcomes. Consequently, these cases have the best performance according to average PI, although, closely followed by day selections in an NGS. An average performance is seen when weeks are selected in a GS. Averaging over the outcomes of GSs and $N G S$ s, day selections show the overall best performance followed by week selections leaving the worst spot for the hour selections. Compared to IS 1, IS 5 overestimates coal and underestimates nuclear capacity. With IS 5 being the most common outcome within the hour selections, it seems that the hours are put together in a way creating too much ramping, forcing the investments to prioritize coal over nuclear. In Section 6.5, we look further into performance values of the different aggregations and here we see that, selecting hours definitely has a higher risk of overestimating ramping values. As pointed out in Section 3, the RL strategies essentially are hour selections even for the day and week element types, which causes these strategies to have the performance of mainly IS 5. On the other hand, aggregation technique SR actually shows to be very consistent independent of element type, since a performance of IS 1 is achieved, even for hour selections. The only other NGs selecting hours is the DX, and here the hour selections performs significantly worse compared to the day and week selections. Averaging the performance of the different selection criteria, the same is seen for the DB and CC techniques. On the other hand, the SC and LC strategies have the worst average performance for week selections, causing the hour selections to have second worst performance.

In conclusion, it is clear that the choice of element type has a high impact on the performance of the aggregation techniques. Especially, with SR being the only exception, one can conclude that for a MILP containing UC, aggregations based on hour selections are not well suitable. On the other hand, taking all analyzed aggregation strategies into account, days seems to be the overall best element to select, which is in agreement with the conclusions of [1]. Consequently, performance consistency over the different element types does not in general seem to be the case. This means that, when an aggregation technique performs well when e.g. days are selected, one can not expect the same good performance when changing the selection to hours or weeks.

\subsubsection{Impact of Selection Criteria on the Aggregation Performance}

To analyze whether some selection criteria perform better than others, the ISs outcomes of the 5 different selection criteria for each of the $4 G S$ aggregation techniques are summarized in Table 13.

For each of the selection criteria, the majority of the single aggregations find IS 1. Random selection shows best performance since the amount of IS 1 outcomes for this is nearly twice as high as for the other selections. Furthermore, only 4 different ISs are seen in the outcomes of random selections while 6 different ISs are seen among the outcomes of the other selection criteria, indicating more performance consistency for random selections. Furthermore, the average PIs of the aggregation strategies (Table 10), show that, all years and all element types considered, the ones based on random selection have best performance for 
Table 12: Distribution of ISs according to elements

\begin{tabular}{|c|c|c|c|c|c|c|c|c|c|c|}
\hline \multicolumn{10}{|c|}{ GS } & VG $(F$ \\
\hline Hours & - & - & - & $1.7 \%$ & $60 \%$ & - & - & $36.7 \%$ & $1.7 \%$ & 4.05 \\
\hline Days & $78.3 \%$ & $1.7 \%$ & - & $13.3 \%$ & - & $5 \%$ & $1.7 \%$ & - & - & 1.08 \\
\hline Weeks & $38.3 \%$ & $41.7 \%$ & $5 \%$ & $13.3 \%$ & - & $1.7 \%$ & - & - & - & 3.30 \\
\hline \multicolumn{11}{|c|}{ NGS } \\
\hline Hours & $33.3 \%$ & - & - & - & $55.6 \%$ & - & - & $11.1 \%$ & - & 3.23 \\
\hline Days & $60 \%$ & - & - & $13.3 \%$ & $26.7 \%$ & - & - & - & - & 2.11 \\
\hline Weeks & $73.3 \%$ & - & - & $6.7 \%$ & $20 \%$ & - & - & - & - & 1.54 \\
\hline
\end{tabular}

Table 13: Distribution of ISs according to selection criteria type

\begin{tabular}{|c|c|c|c|c|c|c|c|c|c|c|c|}
\hline Criteria & IS 1 & IS 2 & IS 3 & \multicolumn{1}{c}{ IS 4 } & \multicolumn{1}{c|}{ IS 5 } & \multicolumn{1}{c|}{ IS 6 } & IS 7 & IS 8 & \multicolumn{1}{c|}{ IS 9 } & \multicolumn{1}{c|}{ AVG(PI) } \\
Mean & $\mathbf{3 6 . 1 \%}$ & $27.8 \%$ & - & $2.8 \%$ & $25 \%$ & - & - & $5.6 \%$ & $2.8 \%$ & 3.40 \\
cMean & $\mathbf{3 0 . 6 \%}$ & $22.2 \%$ & - & $8.3 \%$ & $16.7 \%$ & $5.6 \%$ & - & $16.7 \%$ & - & 3.00 \\
Min & $\mathbf{3 6 . 1 \%}$ & $22.2 \%$ & - & $5.6 \%$ & $19.4 \%$ & $5.6 \%$ & - & $11.1 \%$ & - & 3.08 \\
Max & $\mathbf{3 3 . 3 \%}$ & - & $8.3 \%$ & $22.2 \%$ & $19.4 \%$ & - & $2.8 \%$ & $19.9 \%$ & - & 2.86 \\
Random & $\mathbf{5 8 . 3 \%}$ & - & - & $8.3 \%$ & $19.4 \%$ & - & - & $13.9 \%$ & - & 1.78 \\
\hline
\end{tabular}

each of the 4 aggregation techniques. The performance of the other selection criteria does not seem to be consistent for the different aggregation techniques. Looking at the selection criteria performance across the aggregation techniques (Table 13), still the random selection outperforms the others. Moreover, as pointed out by [24] and [28], representing a group by average values results in underestimation of production and overestimation of total system costs. Within the different aggregation techniques, our results do not indicate that the mean selection criterion has consistently worse performance compared to e.g. selection of minimum or maximum elements. However, looking at the average performance across the 4 techniques, the selection of mean elements has the overall worst performance.

To compare the sensitivity of the different aggregation techniques to the choice of selection criterion, Table 10 shows the average performance over all selection criteria. It is seen that the DB strategy has the overall worst performance while the LC has the overall best. When selecting elements in the DB approach, time chronology has to be respected and consequently less similarity within the groups is achieved. Hence, more diversity is seen among the outcomes of the DB approach for different selection criteria leading to the indication that the choice of selection criterion have higher impact on techniques providing less group similarity. In the following we study whether such a correlation actually can be confirmed.

\section{Similarity Ratio Definition}

Inspired by the $k$-means cluster technique, we define the within group similarity measure based on the sum of square distances. The idea is that the total amount of variability $(T)$ can be measured as the sum of squared distances from each hour, of each element, to the average of that hour, over all elements in the set. Essentially, this can then be divided into within group similarity $(W)$ and between group similarity $(B)$. $W$ is defined as the sum of squared distances from each hour of a single element to the group average of that hour. $B$ is defined as the sum of squared distances between group average hours and average hour over all elements. If we assume that a grouping $G$ consists of $g$ groups, each containing $e$ elements, consisting of $t$ 
hours, the three measures can be defined as follows

$$
\begin{gathered}
T=\sum_{g \in G} \sum_{e \in g} \sum_{t \in e}\left(x_{t, e, g}-\bar{x}_{t}\right)^{2} \\
W=\sum_{g \in G} \sum_{e \in g} \sum_{t \in e}\left(x_{t, e, g}-\bar{x}_{t, g}\right)^{2} \\
B=\sum_{g \in G}|g| \sum_{t \in e}\left(\bar{x}_{t, g}-\bar{x}_{t}\right)^{2}
\end{gathered}
$$

Here $x_{t, e, g}$ is the value of hour $t$ in element $e$ belonging to group $g$. Next, $\bar{x}_{t}$ is the average of hour $t$ over all elements and $\bar{x}_{t, g}$ is the average of hour $t$ of all group elements in group $g$. From the above definitions, a high similarity within the groups would imply large $B$ and small $W$ values. Having the relation $T=W+B$ we define the ratio

$$
R=\frac{B}{T}
$$

which, for higher similarity grouping, should approach 1 . Basically, $R$ describes the percentage of the total variance in the data set explained by the grouping, and by that, how much variance that is expected to be covered in a time series constructed by selecting one element from each group.

\section{Impact of Similarity Ratios on the Performance Consistency}

For the $4 G S$ s considered in this analysis we calculate the ratio $R$. Since each strategy is performed for 3 years and for 3 element types we get the 36 group ratios seen in Table 14 .

Table 14: Group ratios $\mathrm{R}$ of the GSs

\begin{tabular}{|c|c|c|c|c|c|c|c|c|c|}
\hline \multicolumn{9}{c}{ Hour } & \multicolumn{3}{c}{ Day } & \multicolumn{4}{c|}{ Week } \\
Strategy & $\mathbf{2 0 1 4}$ & $\mathbf{2 0 1 5}$ & $\mathbf{2 0 1 6}$ & $\mathbf{2 0 1 4}$ & $\mathbf{2 0 1 5}$ & $\mathbf{2 0 1 6}$ & $\mathbf{2 0 1 4}$ & $\mathbf{2 0 1 5}$ & $\mathbf{2 0 1 6}$ \\
DB & 0.84 & 0.84 & 0.85 & 0.13 & 0.11 & 0.18 & 0.06 & 0.05 & 0.05 \\
SC & $\sim 1$ & $\sim 1$ & $\sim 1$ & 0.92 & 0.91 & 0.92 & 0.40 & 0.38 & 0.42 \\
CC & $\sim 1$ & $\sim 1$ & $\sim 1$ & 0.60 & 0.61 & 0.85 & 0.24 & 0.23 & 0.25 \\
LC & 0.90 & 0.90 & 0.90 & 0.70 & 0.88 & 0.84 & 0.16 & 0.22 & 0.23 \\
\hline
\end{tabular}

As expected, independently of the elements to be selected, the DB approach achieve groups having much larger within group dispersion compared to the groups achieved from clustering approaches. Moreover, from the ratios of the CC and the LC approach, it seems that the nested clustering technique decreases the within group similarity. In general for all the grouping techniques, it is seen that the similarity within the groups increases as smaller elements are grouped. Consequently, for single hours, even when time chronology is respected, a high similarity within the groups is actually achieved.

To analyze the similarities in relation to the model performance diversity, we recall that each of the 36 groupings leads to an aggregated problem having one of the 9 ISs as solution. Each grouping is assigned the number of different ISs seen among the 5 aggregated solutions and the results are seen in Table 25 in Appendix D. Plotting the group ratios against the amount of different ISs gives the relation visualized in Figure 3.

With only 36 data points based on only 4 GSs, a clear conclusion cannot be drawn. However, the distribution of the points in Figure 3 still suggests more consistency in the investment strategies when 


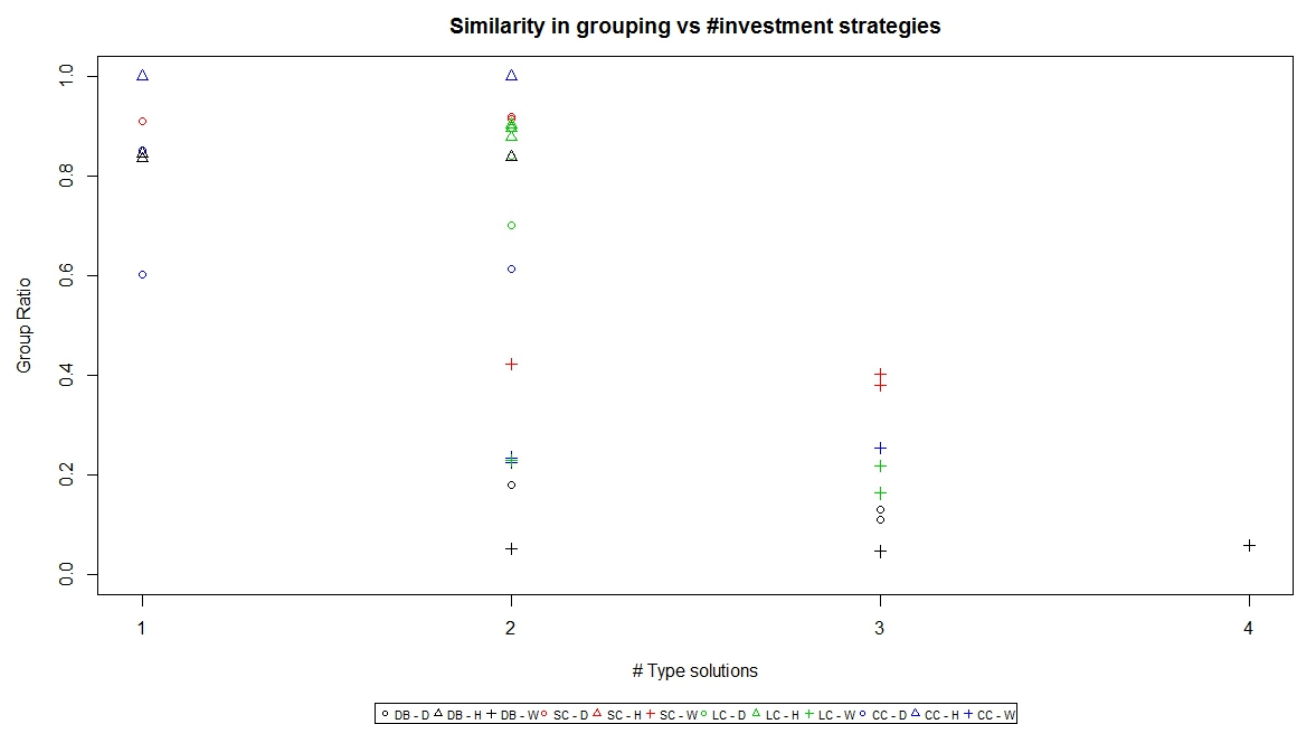

Fig. 3: Similarity ratio of groupings in relation to different ISs observed

selections are made from groups having higher within group similarity, which indicates a confirmation of the suggested correlation.

Returning to the performances of the GSs in Table 10, we see that the random selection of the DB has the worst performance of all random selections, which could indicate that respecting time chronology might not necessarily lead to better model performance. However, the fact that the DB has the worst within group similarity could also be a reason for the bad performance. Usually, when cluster analysis is applied, the aim is to maximize dissimilarities between clusters, resulting in a selection from each cluster describing as much of the variance of the original instance as possible. In the light of time aggregation, it is expected that the more variation of the original time series that is covered in the aggregation, the more precise investment choices can be made, based on the aggregated problem. Assuming that higher similarity within the groups ensure more of the variation to be captured in the aggregated time series, one would expect higher similarity within the groups to result in better performance of the resulting aggregation. To study whether the performance of the aggregation technique is correlated to the group similarity, the average PI over all selection criteria, for each element type and for each year, is plotted with respect to the group similarity in Figure 4. Rather than seeing an overall correlation where higher group similarity gives lower average PI, we see the results being highly dependent on the element type selected. This is also in agreement with our earlier results showing that group similarity increases as element size decreases. While hour selections are clustered in the right upper part having highest similarity, but semi good to bad performance, the week selections are found primarily in the middle lower part having low similarity and average performance. The day selections are grouped in the upper left corner providing the best trade-off between group similarity and model validation performance. A clear exception is the day selection based on the DB approach, which have significantly worse group similarity but only slightly worse model validation performance compared to the rest of the day selections. Now, analyzing these results in the light of the suggested correlation, the conclusion is that group similarity itself is not securing a good model performance. For none of the three element types, the single aggregation, having the best performance, is also having highest within group similarity. Especially for hour selections, but in fact for all types, it is seen that aggregations based on 
groupings with similar within group similarity show very different model validation performance and vice versa.

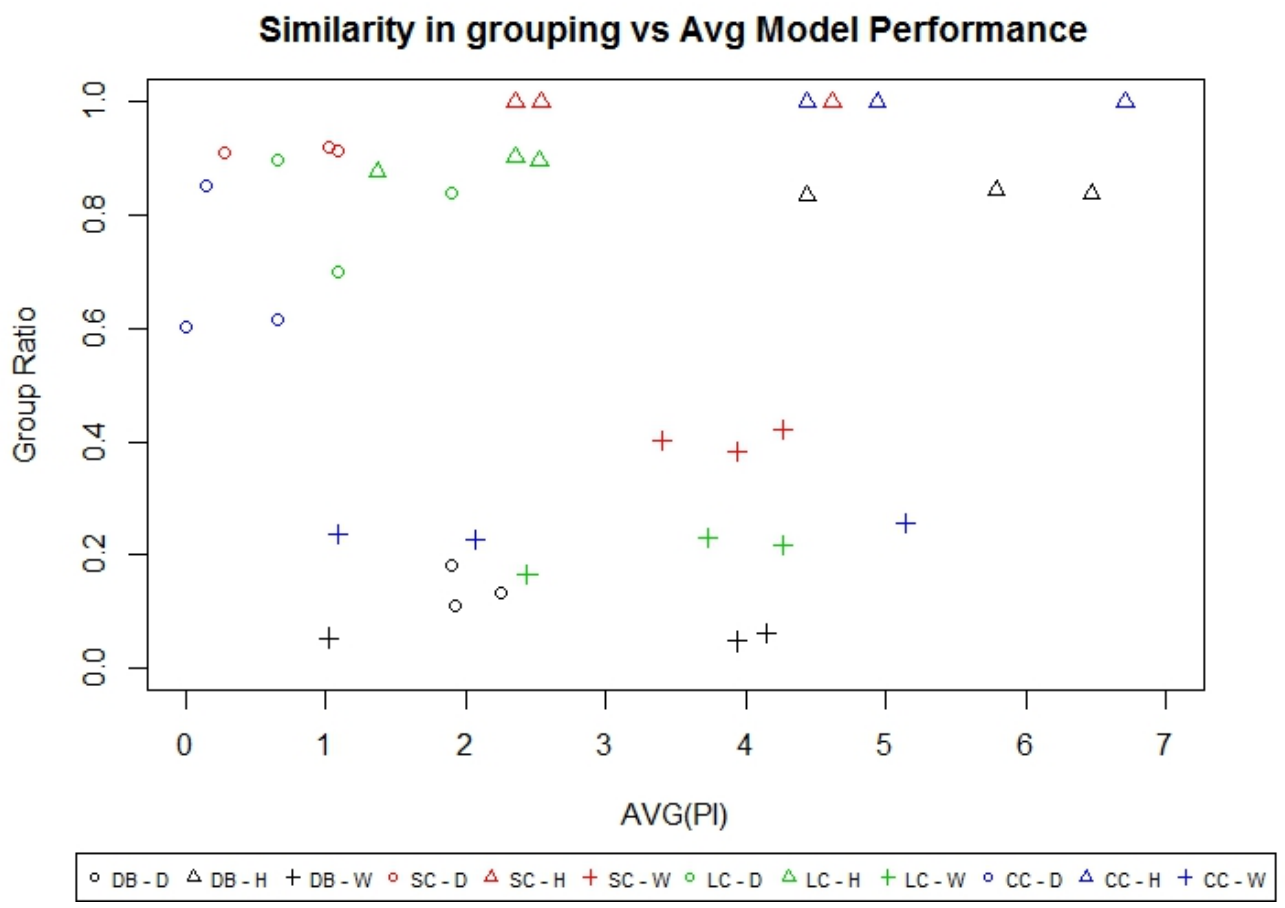

Fig. 4: Similarity ratio of groupings in relation to performance of the technique averaged over all selection criteria

\section{Concluding words on Similarity Ratio in Aggregation Techniques}

From these results we conclude that developing aggregation techniques with an aim of securing high within group similarity is beneficial in the sense that the choice of selection criteria has less impact on the performance of the resulting aggregated problem. However, high within group similarity does not ensure that the resulting aggregated problem replicates the original problem solution.

Another property, that one would expect of a good aggregation strategy, is performance consistency for different instances. Therefore, before moving to the comparison of the single aggregation performances, we shortly look into whether the aggregation techniques are robust to different data sets.

\subsubsection{Performance consistency for different Instances}

From Table 10 and Table 11 it is seen that, for the hour selections, $64 \%$ of the aggregations find the same IS for all years. Selecting days, this number is $50 \%$ and for weeks it is $36 \%$. However, these numbers are heavily affected by some of the $G S$ s being really bad regarding yearly performance consistency, especially when either days or weeks are selected. Of the GS s selecting minimum and maximum elements, only $25 \%$ 
and $33 \%$ of the strategies respectively, show performance consistency over different years. For Mean, cMean and Random selections these numbers are 58\%, 50\% and $75 \%$ respectively. This indicates that aggregation robustness to different instances is highly dependent on the selection criteria. Since we concluded the selection of a random element to have the overall best performance we now disregard the techniques based on the other selection criteria. It is then seen that $71 \%$ of the hour selections find the same IS for the 3 years. For day selections this number is $67 \%$ while it is $63 \%$ for week selections. In total, $67 \%$ of all strategies are consistent in performance for different years. This indicates that, for the selection of best performing aggregation strategies analyzed (Table 15), a general consistency in performance is seen for different instances.

Additionally, the diversity of ISs seen for each of the 3 years indicates a relation between aggregation performance consistency and wind availability in the year. As mentioned earlier, 2015 is considered a wind rich year. Due to this having all 9 ISs among its aggregated solutions, it is the most diversified year regarding aggregation performance. Consequently, our results indicate that aggregation robustness to different instances not only depends on the aggregation technique itself, but also depends on the wind availability, in the sense that aggregation becomes harder when more wind is available.

\subsubsection{Comparison of Aggregation Technique Performances}

Since we already concluded most of the GSs to have worse performance compared to the NGs, and the random selection criterion to have the overall best performance, we limit the aggregation technique comparison to cover the techniques summarized in Table 15.

Table 15: Resulting ISs of the GS (best-off) and $N G S \mathrm{~s}$

\begin{tabular}{|c|c|c|c|c|c|c|c|c|c|c|c|c|c|}
\hline \multirow[b]{2}{*}{ Strategy } & \multicolumn{4}{|c|}{ Hour } & \multicolumn{4}{|c|}{ Day } & \multicolumn{4}{|c|}{ Week } & \multirow[b]{2}{*}{ AVG PI } \\
\hline & 2014 & 2015 & 2016 & AVG & 2014 & 2015 & 2016 & AVG & 2014 & 2015 & 2016 & AVG & \\
\hline DX & IS 5 & IS 5 & IS 5 & 5.65 & IS 1 & IS 1 & IS 1 & 0.14 & IS 1 & IS 1 & IS 1 & 0.14 & 1.98 \\
\hline SR & IS 1 & IS 1 & IS 1 & 0.14 & IS 1 & IS 1 & IS 4 & 1.61 & IS 1 & IS 1 & IS 1 & 0.14 & 0.63 \\
\hline $\mathrm{RL}$ & IS 8 & IS 5 & IS 5 & 3.91 & IS 5 & IS 5 & IS 5 & 5.65 & IS 5 & IS 5 & IS 5 & 5.65 & 5.07 \\
\hline ES & - & - & - & - & - & - & - & - & IS 1 & IS 1 & IS 4 & 1.61 & 1.61 \\
\hline OA & - & - & - & - & IS 1 & IS 1 & IS 4 & 1.61 & - & - & - & - & 1.61 \\
\hline OS & - & - & - & - & IS 1 & IS 5 & IS 1 & 1.53 & IS 1 & IS 1 & IS 1 & 0.14 & 0.84 \\
\hline DB_Random & IS 5 & IS 5 & IS 5 & 5.65 & IS 1 & IS 1 & IS 1 & 0.14 & IS 4 & IS 4 & IS 1 & 3.04 & 2.94 \\
\hline SC_Random & IS 5 & IS 8 & IS 8 & 3.16 & IS 1 & IS 1 & IS 1 & 0.14 & IS 1 & IS 1 & IS 1 & 0.14 & 1.15 \\
\hline CC_Random & IS 5 & IS 5 & IS 5 & 5.65 & IS 1 & IS 1 & IS 1 & 0.14 & IS 1 & IS 1 & IS 4 & 1.61 & 2.47 \\
\hline LC_Random & IS 8 & IS 8 & IS 8 & 1.42 & IS 1 & IS 1 & IS 1 & 0.14 & IS 1 & IS 1 & IS 1 & 0.14 & 0.57 \\
\hline
\end{tabular}

In this selection of techniques, each of the 3 years only cover 3 different ISs, and overall only 4 of the 9 different ISs appear. This shows that, by selecting the best aggregation techniques, we have achieved high performance consistency with respect to different instances, and in general achieved more similar performances among the different techniques. Taking all elements into account, $59 \%$ of the single aggregations have an IS 1 performance, while disregarding hour selections makes this number $76 \%$. We can therefore conclude, that an overall good performance is seen for the selected aggregation techniques. Still, significant differences in performances appears among the different techniques. The worst performing aggregation strategy is the RL due to the fact that it is based on hourly selection for each element type. As discussed in Section 6.2.1, a selection of hours seems to lack important within element chronology, which cannot be captured by reconstructing chronology. The overall best performance is seen for the LC with random selections, closely followed by the SR approach. The SR approach consistently finds IS 1 with a single exception being day selections of year 2016. For the LC approach, the performance of the hour selections 
is the second best IS, namely 8, while the rest is found to be IS 1 . From the overall average PI it is seen that the LC approach has a significant better performance compared to the CC approach. With the only difference between the two approaches being that the LC also considers correlation of the elements, it can be concluded that aggregation techniques benefit from selecting elements both according to residual level and correlation. Moreover, with the random selection criteria being the overall best performing, and considering that the LC and SR approaches both include a lot of randomness, it can also be concluded that aggregation techniques seem to benefit from the inclusion of randomness.

Looking at the specific element types we see that for day selections, the DX approaches and all GSs perform best and equally well, while for week selections the aggregation strategies DX, SR, OS, SC and LC have best and equally good performance. Now, comparing the performance of the strategies, in the light of the complexity associated to the strategies, we see that for day or week selections both the DX, the SC and the LC have the exact same performance. All of these have fairly low running times (see Table 2), but regarding implementation complexity the DX strategy is the simplest one, and furthermore a single cluster is simpler compared to a nested cluster approach. Looking at the OS and the OA strategies, these are probably the most demanding approaches, implementation-wise, and even though the aggregations show good results, they do not perform significantly better than the more simple aggregations. As a last remark, the RL is the most complex heuristic selection strategy included in this analysis, and the results indicate that it is the overall worst performing strategy. This gives us a clear basis for concluding that simple aggregation strategies can lead to aggregated problems replicating the original problem solution as good as aggregation strategies having higher complexity.

Although the problems might not be fully comparable, a similar conclusion is seen within the theory of Neural Networks [10], where higher model complexity potentially leads to higher errors. Essentially, the inclusion of too much information in the models causes an overfitting which leads to the worse performance. Returning to the results of this paper, one could imaging that the inclusion of too much information of the original time series in the selection, potentially could mislead the search, or the extra information could simply be irrelevant, causing similar performances despite complexity differences. Illustrating this by an example, the DX approach uses no information of original time series and still performs better than the OS approach where the search is guided by several values of the original time series.

\subsection{Data Validation vs. Model Validation}

Next, we study whether a good performance according to our model validation also provides good performance according to the commonly used data validation. Recall that the data validation is the ability of the aggregated time series to replicate the RLCD of the original time series. This is measured according to the NRMSE between the aggregated and the original RLDCs. The NRMSEs for the selection of the best performing strategies are seen in Table 16 where the color of the number indicates the IS achieved for the corresponding single aggregation. To analyze the effect of having a strategical selection (using the aggregation techniques) rather than having a blind selection, we calculated minimum, maximum and average NRMSEs of a span of 1000 blind selections (selecting randomly) of the specific element, as seen in the last rows of the table.

In general, based on the results in Table 16, it can be concluded that the smaller elements one selects, the better the selected data replicates the original RLDC. Recalling the performance challenges of the aggregations based on hour selections, this conclusion already now indicates a complication in a possible correlation between data and model validation. Sticking to the hour selections, the worst performance according to the NRMSE is seen for the SR strategy, which is the second best performing strategy according to model results. Furthermore, according to the NRMSE, the RL approach performs particularly well within each element category, having one of the lowest average NRMSEs, while it, according to model performance, has a significantly worse performance compared to the other strategies. Moreover, from the values in Table 
Table 16: NRMSE of the GSs (best-off) and NGSs with the IS as coloring

\begin{tabular}{|c|c|c|c|c|c|c|c|c|c|c|}
\hline \multicolumn{9}{c}{ Hour } & \multicolumn{9}{c|}{ Day } & \multicolumn{7}{c|}{ Week } \\
\multicolumn{1}{|c|}{ Strategy } & $\mathbf{2 0 1 4}$ & $\mathbf{2 0 1 5}$ & $\mathbf{2 0 1 6}$ & $\mathbf{2 0 1 4}$ & $\mathbf{2 0 1 5}$ & $\mathbf{2 0 1 6}$ & $\mathbf{2 0 1 4}$ & $\mathbf{2 0 1 5}$ & $\mathbf{2 0 1 6}$ & AVG NRMSE \\
\hline DX & 0.66 & 0.75 & 0.61 & $\mathbf{3 . 2 0}$ & $\mathbf{8 . 0 7}$ & $\mathbf{1 . 7 8}$ & $\mathbf{3 . 3 8}$ & $\mathbf{3 . 2 6}$ & $\mathbf{6 . 1 1}$ & 3.09 \\
SR & $\mathbf{1 . 5 8}$ & $\mathbf{2 . 3 7}$ & $\mathbf{2 . 4 5}$ & $\mathbf{0 . 8 7}$ & $\mathbf{2 . 4 4}$ & 2.75 & $\mathbf{2 . 6 4}$ & $\mathbf{1 . 3 4}$ & $\mathbf{0 . 9 6}$ & 1.93 \\
RL & 0.22 & 0.20 & 0.19 & $\mathbf{1 . 3 3}$ & $\mathbf{1 . 4 4}$ & $\mathbf{1 . 3 4}$ & 0.36 & 0.36 & 0.37 & 0.65 \\
ES & - & - & - & - & - & - & $\mathbf{0 . 5 9}$ & $\mathbf{0 . 5 9}$ & $\mathbf{0 . 5 4}$ & 0.57 \\
OA & - & - & - & $\mathbf{2 . 1 3}$ & $\mathbf{0 . 9 2}$ & $\mathbf{1 . 9 7}$ & - & - & - & 1.67 \\
OS & - & - & - & $\mathbf{1 0 . 2 5}$ & $\mathbf{1 4 . 3 2}$ & $\mathbf{1 1 . 4 8}$ & $\mathbf{1 . 4 6}$ & $\mathbf{2 . 0 3}$ & $\mathbf{3 . 2 5}$ & 7.13 \\
DB_Random & 0.77 & 0.75 & 0.72 & $\mathbf{1 2 . 3 2}$ & $\mathbf{4 . 5 0}$ & $\mathbf{4 . 5 0}$ & $\mathbf{7 . 1 1}$ & $\mathbf{1 . 4 8}$ & $\mathbf{7 . 1 1}$ & 4.36 \\
SC_Random & 0.09 & 0.08 & 0.09 & $\mathbf{1 . 1 5}$ & $\mathbf{1 . 0 4}$ & $\mathbf{1 . 1 8}$ & $\mathbf{2 . 2 5}$ & $\mathbf{1 . 9 6}$ & $\mathbf{5 . 4 4}$ & 1.48 \\
CC_Random & 0.08 & 0.05 & 0.07 & $\mathbf{1 . 2 7}$ & $\mathbf{2 . 3 6}$ & $\mathbf{1 . 0 4}$ & $\mathbf{3 . 9 7}$ & $\mathbf{4 . 9 8}$ & $\mathbf{7 . 5 8}$ & 2.37 \\
LC_Random & 0.53 & 0.54 & 0.62 & $\mathbf{1 . 9 2}$ & $\mathbf{0 . 8 9}$ & $\mathbf{2 . 0 6}$ & $\mathbf{2 . 1 2}$ & $\mathbf{3 . 7 1}$ & $\mathbf{4 . 3 4}$ & 1.86 \\
\hline Blind (Max) & 2.29 & 2.57 & 2.45 & 9.58 & 9.82 & 936 & 11.94 & 13.30 & 13.87 & 7.26 \\
Blind (Mean) & 1.00 & 1.04 & 1.00 & 3.08 & 3.06 & 3.24 & 4.11 & 4.25 & 5.02 & 2.87 \\
Blind (Min) & 0.42 & 0.46 & 0.46 & 0.83 & 0.81 & 0.76 & 1.02 & 0.80 & 0.99 & 0.73 \\
\hline
\end{tabular}

15 and Table 16, multiple counter examples of a possible correlation between NRMSE and model validation performance can be seen. Selecting days from the 2016 instance, aggregation strategy OA and SR have a much lower NRMSE compared to the OS approach. Still, the aggregated problem based on the SR and OA aggregated data find IS 4 while the problem based on the OS aggregated data finds IS 1. A similar example is found when weeks are selected from the 2015 instance. Here the SC based aggregated problem has worse model validation performance compared to the CC based aggregated problem, even though the SC aggregation has a lower NRMSE value.

Comparing the strategical selections to the blind selections with respect to element type, it is seen that for hour selections, the majority of the strategies perform significantly better than the average and even the best performing blind selection. On average, selecting hours strategically has an average NRMSE of 0.64 , while a blind selection on average has an NRMSE of 1.01. Contrary, looking at day selections, selecting blindly seems to be better than selecting strategically, since a random day selection on average has an NRMSE of 3.13 while a strategical selection on average has an NRMSE of 3.65. For week selection these numbers again favor the strategical selection with an average NRMSE of 2.94 against 4.46 for the blind selection. Consequently, if it was to be concluded that a better replication of the original instance lead to better performance of the aggregated problem, aggregating according to days should preferably be done as a blind selection. However, according to the model validation performance (Table 15) strategically selecting elements has an average PI of 3.65 for hours, 1.23 for days and 1.40 for weeks, indicating that the strategical selection of days has the overall best chances of good performance according to model validation. This clearly contradicts with the bad performance of strategical day selection according to data validation.

To visualize these results, we plot the model validation performance index as a function of the NRMSE value for each of the single aggregations as seen in Figure 5. The specific single aggregations are not marked in the graph, only whether the aggregation is based on hour, day or week selection. A regression line is added to the plot to visualize a possible correlation. The line shows a decreasing tendency but with a corresponding $R^{2}$ value of 0.0099 we can conclude that the points are very poorly described by this regression. With single aggregations having good model validation performance for both small and large NRMSEs, and good data validation performance for both small and large PIs, there is no indication of that a good performance within one of the criteria ensures a good performance in the other.

However, due to our conclusion about aggregation performance not being consistent for different element types, it might not be fair to look for a correlation among all the points. Instead we split the points 


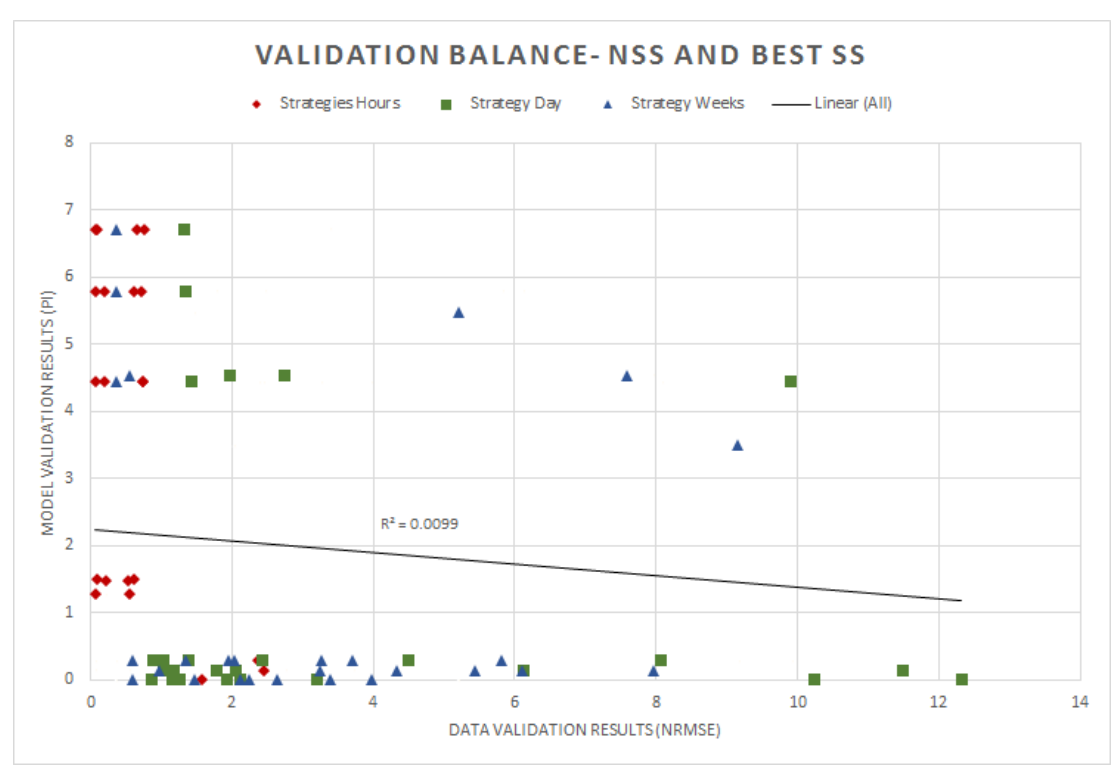

Fig. 5: NRMSE and PI relation of all strategies. The line shows the best linear regression between the PI and the NRMSE with the $R^{2}$ value being the quality measure of the regression.

according to element types and look for a correlation within each type, see Figure 6a, Figure $6 \mathrm{~b}$ and Figure 6c. For the hour selections (Figure 6a), again a decreasing regression line with a very low $R^{2}$ value is seen. The negative slope is caused by the 3 points having high NRMSEs but good model validation performance. Disregarding these, an increasing regression line is found with an $R^{2}$ value of 0.0072 . However, the disregarded points correspond to the SR strategy, which is the strategy having the overall second best performance. Consequently, the results again indicate that better data validation performance does not ensure better model validation performance. The day selections (Figure 6b) show similar results to the hour selections with a bit more diversity in the data validation performance. Again, outliers, having high error according to data similarity and good model validation performance, cause a decreasing regression with an $R^{2}$ value of 0.0096 . These outliers primarily correspond to the DB strategy, which, for day selection, has the best possible performance for each year. Also in the week selections (Figure 6c), the DB strategy causes outliers in 2 out of 3 years, but without these, the points still show no indication of a correlation but rather just different model validation performance for low NRMSEs as well as low and high NRMSEs for similarly good model validation performance.

Although, our analysis indicates consistency in model validation performance among different instances, it does not seem that the same consistency is seen for data validation performance. Therefore, as a last try, we look for a possible correlation between the average NRMSE and the average PI over the different years. The results are visualized in Appendix D, Figure 7 for all element types and in Figure 8 for the single element types. In short, the same tendency is seen as before, where tendency lines with negative slope and low $R^{2}$ values dominate the results. The conclusion is again that good model validation performance can appear for any data validation performance and vice versa. 


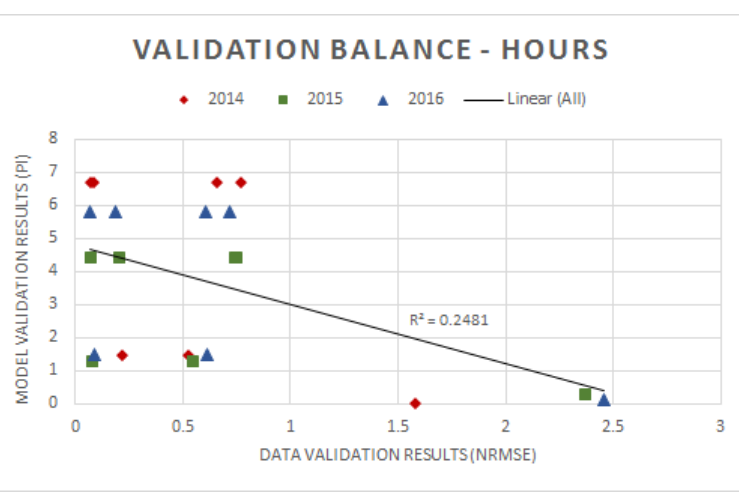

(a) Hours

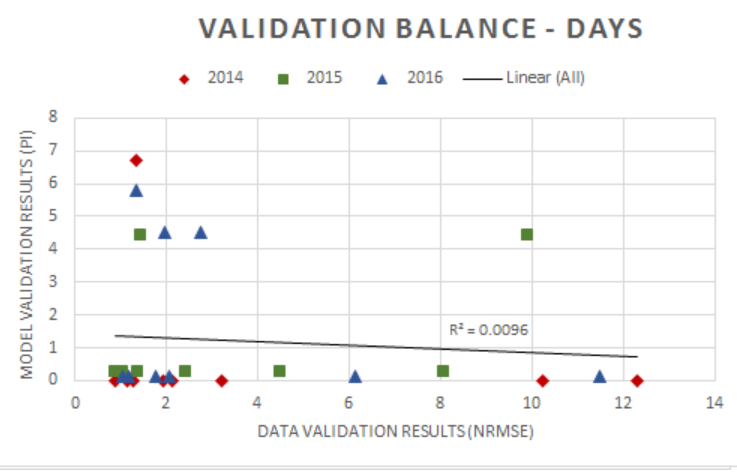

(b) Days

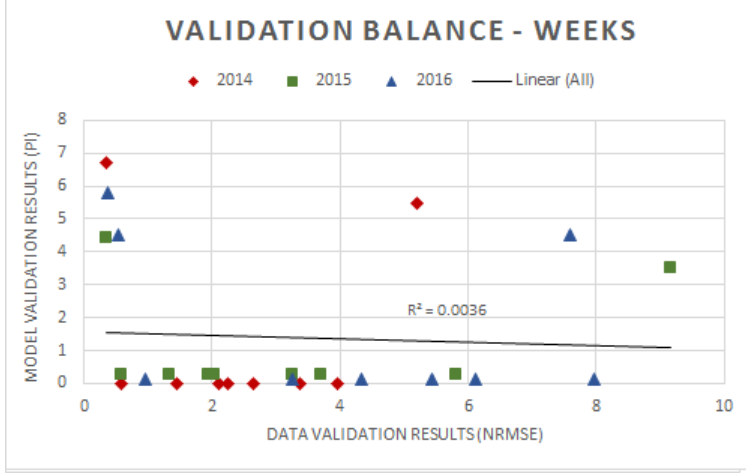

(c) Weeks

Fig. 6: NRMSE and PI relation of all strategies, but divided into hour, days, and week elements

\subsection{Solution time comparison}

The purpose of aggregating energy problems is to achieve tractability or to reduce solution times. Hence, the solution time of the aggregated problems is crucial to analyze. Table 17 presents the solution times achieved for the different aggregated problems for each of the years.

Table 17: Solution times for aggregated problems based on best-off aggregations (CPLEX times)

\begin{tabular}{|c|c|c|c|c|c|c|c|c|c|c|c|c|c|}
\hline \multirow[b]{2}{*}{ Strategy } & \multicolumn{4}{|c|}{ Hour } & \multicolumn{4}{|c|}{ Day } & \multicolumn{4}{|c|}{ Week } & \multirow[b]{2}{*}{ AVG Time } \\
\hline & 2014 & 2015 & 2016 & AVG & 2014 & 2015 & 2016 & AVG & 2014 & 2015 & 2016 & AVG & \\
\hline DX & 38.78 & 97.37 & 51.65 & 62.6 & 66.64 & 141.05 & 119.16 & 109.0 & 79.5 & 94.52 & 78.23 & 84.1 & 85.31 \\
\hline SR & 77.06 & 78.21 & 92.93 & 82.7 & 65.42 & 157.81 & 89.07 & 104.1 & 73.34 & 36.03 & 102.67 & 70.7 & 87.22 \\
\hline RL & 119.99 & 327.17 & 47.79 & 165.0 & 37.36 & 38.46 & 25.11 & 33.6 & 72.63 & 49.68 & 44.09 & 55.5 & 70.42 \\
\hline ES & - & - & - & - & - & - & - & - & 99.38 & 94.48 & 62.6 & 85.5 & 85.49 \\
\hline $\mathrm{OA}$ & - & - & - & - & 100.27 & 84.56 & 26.44 & 70.4 & - & - & - & - & 70.4 \\
\hline OS & - & - & - & - & 35.18 & 36.86 & 87.52 & 53.2 & 101.68 & 112.25 & 78.4 & 97.4 & 72.15 \\
\hline DB_Random & 29.3 & 50.85 & 66.27 & 48.8 & 47.72 & 77.88 & 84.56 & 70.1 & 97.19 & 73.34 & 212.33 & 127.6 & 78.03 \\
\hline SC_Random & 65.15 & 2460.95 & 653.85 & 1060.0 & 60.97 & 45.66 & 71.03 & 59.2 & 76.35 & 60.4 & 96.11 & 77.6 & 428.15 \\
\hline CC_Random & 50.39 & 57.88 & 80.33 & 62.9 & 64.16 & 94.24 & 95.19 & 84.5 & 77.52 & 92.22 & 85.62 & 85.1 & 76.81 \\
\hline LC_Random & 66.92 & 1064.49 & 232.47 & 454.6 & 61.17 & 74.76 & 56.2 & 64.0 & 77.21 & 91.32 & 114.09 & 94.2 & 214.3 \\
\hline
\end{tabular}


It is seen that most of the aggregated problems are solved within a timeframe of approximately 25 sec - 3.5 minutes with a few exceptions being the RL (week, 2015), the SC (Hour, 2015 \& 2016) and the LC (Hour, 2015) aggregations. Of these, the solution time of the RL approach is still under 6 min and is, therefore, only a minor deviation compared to the SC and LC strategies where the solution times are 11 min, $18 \mathrm{~min}$ and $41 \mathrm{~min}$ respectively. The largest solution times are seen for year 2015 indicating that this is the most challenging year. Furthermore, it can be concluded that not only the size of the aggregated instance affects the solution time, but also very much the composition of the aggregated instance. All of the diverging solution times are based on hourly selections making this the most challenging element type to select. This is likely due to the challenge of putting the hours together in a smart way. From our results it is seen that this also depends on the original time series since the solution times of the 2014 aggregations never exceeds 2 min independent of element type and year. In conclusion, whenever aggregation is applied, unlucky selections can be made, making the gains according to solution time less attractive. However, recalling the solution times of the original problems in Table 6 , a solution time of 41 minutes is still a $50 \%$ reduction, and most of the other aggregations find investment strategies $98 \%$ faster compared to the non-aggregated problem.

In the model validation of the aggregated problems, we first solved the aggregated problem and then fixed the resulting investment choices in the non-aggregated problem (Procedure 2). An interesting point is that, by fixing the investment choices, the solution time of the non-aggregated problem is reduced significantly. By only fixing 28 binary variables the non-aggregated problem is solved in 1-5 min dependent on the aggregation strategy (Table 26, Appendix D). With the original problem, with non-fixed investments, having solution times of 71-95 minutes dependent on the year, this illustrates how much more complex the problem becomes when investment decisions are included.

\subsection{Performance values of Aggregated data}

Although our analysis covered 219 single aggregations, only 9 different ISs were seen as results. It is therefore interesting to see how different the aggregated time series are and if there is a correlation between different performance values of the data and the performance of the aggregated problem. To enlighten this, we study a set of performance values consisting of the average, minimum and maximum values of both the level and ramping values of the aggregated time series. Also, the variance of the aggregated time series is analyzed. The values are compared to the same performance values of the original time series and the results are seen in Table 18, 19 and 20. All values are percentage differences to the values of the original series, except for the average ramping values. Since these are close to zero, they are stated as actual differences.

Both for the ES and the OA approach, the level values of the original time series seem to be captured well while the minimum and maximum ramping values are poorly captured. Regarding the variance, the ES approach is almost capturing this perfectly unlike the OA aggregated data. For both approaches, IS 1 is found except in year 2016, where a clear deviation in performance values is a significant underestimation of minimum and maximum ramping values. The probably best overall capture of level and ramping values is seen for the OS approach, and regarding the model performance of these aggregations, only daily selections of 2015 did not achieve IS 1 . The only clear deviation from this strategy to the others is that the variance is very overestimated.

For the selected DB, SC, CC and LC aggregation strategies and for the DX aggregations almost only IS 1 is achieved whenever days and weeks are selected, while all the hour selections lead to worse performance. The hour selections capture the level values well but overestimate both minimum and maximum ramping values and the variances. However, for some of the approaches such as the DB, the overestimation of the hourly aggregations is not significantly worse compared to the overestimation seen for day selections.

The aggregations based on the RL approach are capturing the levels of the original time series almost perfectly, and the same holds for the variation, with the day selections as exceptions. Regarding ramping 
values, also here selecting days is causing the highest deviations from the values of the original time series. Regarding performance, all single aggregations, except for hour selections of year 2014, achieve IS 5. The hour selections of 2014 find IS 8, which actually is a better performance compared to IS 5, but the performance values do not indicate a significant difference between the 2014-hour points and the others. Comparing the performance values of the RL with the performance values of the SR approach, it is seen that the aggregations based on SR are capturing minimum and maximum levels significantly worse than the aggregations based on RL. Furthermore, the rampings and the variance are captured to somewhat same degree in the two approaches. A clear difference between the performance values of the RL and the SR approach is that the aggregations based on the SR almost purely underestimate the variance while the RL aggregations overestimate the variance. However, looking at week selections of year 2016, very similar performance values are seen for the RL and the SR aggregations, and yet still the second worst performing IS and the best performing IS are found for the two aggregations. It therefore seems that the performance values of the aggregations are not giving any indication of how the aggregated problem will perform or, more importantly, that capturing the performance values of the original time series does not ensure better performance of the aggregated problem.

In conclusion, this analysis show that the very similar performance among the aggregation techniques definitely is not caused by similarity among the aggregated time series, since very different aggregated time series are achieved from the different aggregation techniques. Furthermore, finding a pattern in the performance values of the aggregated instances and the performance of the corresponding aggregated problem would be of great value to the development of aggregation techniques. However, despite a variety of approaches, selection types, and original time series, such a pattern has not been found. Still, it is valuable for the development of aggregation techniques to know, that a good capture of the performance values of the original time series, not necessarily ensure good aggregated model performance, and that very different time series can provide the same good model validation performance. 
Table 18: Ability of the aggregated time series to replicate performance values of the original time series.

\begin{tabular}{|c|c|c|c|c|c|c|c|c|c|c|c|}
\hline Statistic & Strategy & 2014 & $\begin{array}{l}\text { Hour } \\
2015\end{array}$ & 2016 & 2014 & $\begin{array}{r}\text { Day } \\
2015\end{array}$ & 2016 & 2014 & $\begin{array}{l}\text { Week } \\
2015\end{array}$ & 2016 & AVG(ABS) \\
\hline \multirow{10}{*}{ Mean (Level) } & $\overline{\mathrm{DX}}$ & -0.43 & -0.23 & -0.45 & 11.27 & 24.00 & -6.45 & 6.23 & 7.76 & 16.74 & 8.17 \\
\hline & SR & 2.72 & -0.90 & 2.07 & 3.30 & -0.42 & -5.67 & 1.42 & -0.85 & -0.77 & 2.01 \\
\hline & RL & -0.20 & -0.21 & -0.18 & 0.50 & -3.40 & -6.46 & -0.62 & -0.70 & -0.61 & 1.43 \\
\hline & $\mathrm{ES}$ & - & - & - & - & - & - & -0.71 & -0.72 & 0.20 & 0.54 \\
\hline & OA & - & - & - & 2.20 & 3.79 & 8.85 & - & - & - & 4.95 \\
\hline & OS & - & - & - & 3.41 & -0.00 & -3.53 & -0.22 & -2.08 & 0.42 & 1.61 \\
\hline & DB_Random & 3.06 & 0.62 & 1.09 & 0.35 & 16.03 & 15.36 & -16.36 & 15.28 & 9.99 & 8.68 \\
\hline & SC_Random & 0.77 & -0.73 & -2.05 & 1.28 & 2.55 & 10.09 & 4.43 & -0.72 & -7.87 & 3.39 \\
\hline & CC_Random & 7.80 & 4.88 & -1.03 & 7.45 & 2.38 & 6.28 & -1.34 & 25.91 & -11.96 & 7.67 \\
\hline & LC_Random & 4.84 & 0.41 & 3.05 & 6.27 & 2.92 & 3.87 & 2.29 & 0.12 & -4.47 & 3.13 \\
\hline \multirow{10}{*}{ Max (Level) } & $\overline{D X}$ & 7.09 & 5.86 & 4.18 & 4.92 & 16.38 & 10.36 & 20.94 & 3.61 & 17.92 & 10.14 \\
\hline & SR & 8.56 & 11.06 & 5.78 & 9.91 & 5.01 & 15.02 & 11.19 & 11.25 & 0 & 8.64 \\
\hline & RL & 0 & 0 & 0 & 0.02 & 0 & -0.02 & 0 & 0 & 0 & 0.00 \\
\hline & $\mathrm{ES}$ & - & - & - & - & - & - & 8.57 & 5.86 & 7.25 & 7.23 \\
\hline & OA & - & - & - & 16.47 & 0 & 8.14 & - & - & - & 8.20 \\
\hline & OS & - & - & - & 0.02 & 0 & -0.02 & 0 & 0.00 & 1.01 & 0.18 \\
\hline & DB_Random & 11.85 & 3.82 & 0 & 0 & 9.15 & 8.55 & 13.17 & 3.61 & 1.01 & 5.68 \\
\hline & SC_Random & 0 & 0 & 0 & 0 & 8.88 & 8.56 & 11.19 & 9.15 & 5.78 & 4.84 \\
\hline & CC_Random & 0 & 0 & 0 & 9.22 & 3.61 & 1.54 & 4.90 & 3.61 & 1.01 & 2.65 \\
\hline & LC_Random & 4.71 & 3.61 & 2.52 & 12.14 & 5.86 & 0 & 4.71 & 6.98 & 3.94 & 4.94 \\
\hline \multirow{10}{*}{ Min (Level) } & $\overline{D X}$ & 27.11 & 9.56 & 0 & 32.57 & 9.93 & 8.46 & 51.48 & 14.63 & 25.47 & 19.91 \\
\hline & SR & 26.97 & 38.98 & 40.44 & 35.68 & 7.28 & 33.00 & 70.89 & 41.79 & 25.47 & 35.61 \\
\hline & RL & 0 & 0 & 0 & 7.66 & 0 & -8.30 & 0 & 0 & 0 & 1.77 \\
\hline & $\mathrm{ES}$ & - & - & - & - & - & - & 15.85 & 4.44 & 27.82 & 16.04 \\
\hline & OA & - & - & - & 11.71 & 25.33 & 12.01 & - & - & - & 16.35 \\
\hline & OS & - & - & - & 7.66 & 0 & -8.30 & 14.81 & 0 & 0 & 5.13 \\
\hline & DB_Random & 12.59 & 0 & 18.09 & 29.64 & 12.43 & 0 & 144.31 & 12.32 & 13.86 & 27.03 \\
\hline & SC_Random & 2.65 & 4.44 & 0 & 48.22 & 9.93 & 11.71 & 26.01 & 0 & 43.35 & 16.26 \\
\hline & CC_Random & 0 & 0 & 2.36 & 39.23 & 17.71 & 11.71 & 33.14 & 12.32 & 29.64 & 16.23 \\
\hline & LC_Random & 2.65 & 2.18 & 2.36 & 41.25 & 7.28 & 49.73 & 15.85 & 11.85 & 29.64 & 18.09 \\
\hline
\end{tabular}


Table 19: Ability of the aggregated time series to replicate performance values of the original time series.

\begin{tabular}{|c|c|c|c|c|c|c|c|c|c|c|c|}
\hline \multirow[b]{2}{*}{ Statistic } & \multirow[b]{2}{*}{ Strategy } & \multicolumn{3}{|c|}{ Hour } & \multicolumn{3}{|c|}{ Day } & \multicolumn{3}{|c|}{ Week } & \multirow[b]{2}{*}{ AVG(ABS) } \\
\hline & & 2014 & 2015 & 2016 & 2014 & 2015 & 2016 & 2014 & 2015 & 2016 & \\
\hline \multirow{10}{*}{ Mean (Ramp) } & DX & -2.44 & -0.75 & -0.37 & 1.66 & 0.43 & 0.65 & 1.09 & 0.39 & 1.07 & 0.98 \\
\hline & SR & -4.40 & 2.40 & 4.09 & 1.73 & -4.79 & -1.02 & -1.25 & -0.75 & 1.85 & 2.48 \\
\hline & RL & 1.73 & -1.08 & 2.82 & 2.66 & -0.22 & 1.04 & 0.88 & 3.04 & 2.29 & 1.75 \\
\hline & ES & - & - & - & - & - & - & 2.33 & -3.5 & -0.96 & 2.26 \\
\hline & $\mathrm{OA}$ & - & - & - & -2.51 & 1.06 & 0.70 & - & - & - & 1.42 \\
\hline & OS & - & - & - & -6.00 & -2.49 & -2.51 & -3.65 & 2.20 & 0.22 & 2.85 \\
\hline & DB_Random & -0.60 & -0.30 & 0.58 & 0.26 & -0.67 & -1.14 & -1.43 & -2.29 & -0.85 & 0.90 \\
\hline & SC_Random & -9.80 & 1.35 & 6.47 & 4.11 & 3.33 & -0.32 & 1.96 & -0.87 & -0.22 & 3.16 \\
\hline & CC_Random & -2.26 & -4.58 & -0.02 & -0.95 & 1.84 & -1.99 & -4.75 & -3.25 & 0.80 & 2.27 \\
\hline & LC_Random & -2.39 & -6.48 & 1.18 & -0.70 & -0.98 & -3.72 & -5.50 & -1.97 & -0.04 & 2.55 \\
\hline \multirow{10}{*}{ Max (Ramp) } & $\overline{D X}$ & -233.24 & -74.79 & -27.39 & 24.16 & 1.91 & -105.88 & -114.18 & 13.71 & 66.39 & 73.52 \\
\hline & SR & 0 & 51.45 & 70.10 & 62.05 & 5.27 & -189.29 & 11.17 & 58.80 & 68.09 & 57.36 \\
\hline & $\mathrm{RL}$ & 10.53 & 59.26 & 71.17 & 10.76 & -75.55 & -104.52 & 4.49 & 4.51 & 59.03 & 44.42 \\
\hline & ES & - & - & - & - & - & - & -57.50 & 8.42 & 72.78 & 46.23 \\
\hline & $\mathrm{OA}$ & - & - & - & -177.24 & -0.35 & 39.70 & - & - & - & 72.43 \\
\hline & OS & - & - & - & 45.10 & 0 & -170.05 & 0 & 0 & 0 & 35.86 \\
\hline & DB_Random & -144.21 & -45.02 & 11.46 & 29.78 & -30.85 & -155.15 & -66.92 & 42.96 & 50.12 & 96.08 \\
\hline & SC_Random & -281.92 & -98.10 & -62.52 & -4.44 & -11.15 & -150.00 & 35.57 & 0 & -0.12 & 71.54 \\
\hline & CC_Random & -231.82 & -106.79 & -62.16 & 6.57 & 1.63 & -68.41 & 0 & 58.30 & 67.20 & 66.99 \\
\hline & LC_Random & -352.88 & -144.19 & -68.11 & 41.43 & -0.08 & 65.30 & -82.11 & -21.57 & 70.10 & 93.97 \\
\hline \multirow{10}{*}{ Min (Ramp) } & $\overline{D X}$ & -398.67 & -74.02 & -2.51 & 15.94 & -26.97 & -264.41 & 24.96 & 21.90 & 27.47 & 95.21 \\
\hline & $\mathrm{SR}$ & 10.12 & 56.56 & 75.70 & 24.94 & -40.31 & -190.72 & -2.82 & 17.52 & 46.24 & 51.66 \\
\hline & RL & -58.82 & 62.39 & 78.78 & 18.16 & -16.82 & -284.53 & -54.11 & 32.97 & 27.62 & 70.47 \\
\hline & $\mathrm{ES}$ & - & - & - & - & - & - & -113.83 & 19.55 & 72.75 & 68.71 \\
\hline & $\mathrm{OA}$ & - & - & - & -192.26 & -25.93 & 8.25 & - & - & - & 75.48 \\
\hline & OS & - & - & - & -19.30 & -71.66 & -332.49 & -166.02 & 0 & 0 & 98.25 \\
\hline & DB_Random & -222.69 & -45.96 & 0 & 43.96 & -53.38 & -244.51 & -28.59 & 65.90 & 2.49 & 78.61 \\
\hline & SC_Random & -497.78 & -136.71 & -56.96 & 2.94 & -47.91 & -318.90 & 7.21 & -66.58 & 17.11 & 128.01 \\
\hline & CC_Random & -464.26 & -118.85 & -39.99 & -15.06 & -41.21 & -288.63 & -272.75 & 65.90 & 69.53 & 152.91 \\
\hline & LC_Random & -449.86 & -149.68 & -58.93 & 36.69 & 0 & -206.43 & -89.97 & 7.47 & 12.31 & 112.37 \\
\hline
\end{tabular}

Table 20: Ability of the aggregated time series to replicate performance values of the original time series.

\begin{tabular}{|c|c|c|c|c|c|c|c|c|c|c|c|}
\hline Statistic & Strategy & 2014 & $\begin{array}{l}\text { Hour } \\
2015\end{array}$ & 2016 & 2014 & $\begin{array}{l}\text { Day } \\
2015\end{array}$ & 2016 & 2014 & $\begin{array}{l}\text { Week } \\
2015\end{array}$ & 2016 & AVG(ABS) \\
\hline \multirow{10}{*}{ Variance } & DX & 3.72 & -3.67 & -2.84 & 4.94 & 19.56 & 3.26 & 23.26 & 4.29 & 16.10 & 9.07 \\
\hline & SR & -1.56 & -12.62 & -12.50 & 6.36 & 1.60 & -8.58 & -11.42 & -0.50 & -0.32 & 5.83 \\
\hline & RL & -0.32 & -0.37 & -0.30 & 2.35 & -6.32 & -15.59 & -0.80 & -0.50 & -0.43 & 3.00 \\
\hline & $\mathrm{ES}$ & - & - & - & - & - & - & 0.90 & -0.18 & -0.23 & 0.44 \\
\hline & OA & - & - & - & 7.36 & -2.67 & -16.16 & - & - & - & 8.73 \\
\hline & OS & - & - & - & -33.49 & -58.31 & -60.56 & -10.92 & 1.50 & -33.38 & 33.03 \\
\hline & DB_Random & -4.19 & -3.87 & -8.07 & -9.44 & -47.47 & -46.72 & 36.54 & -17.57 & -71.84 & 27.30 \\
\hline & SC_Random & -8.96 & -2.94 & -2.45 & -26.45 & -4.30 & -7.19 & 11.54 & -16.29 & 14.13 & 10.47 \\
\hline & CC_Random & -18.25 & -13.85 & -17.80 & -9.38 & -10.86 & -30.01 & -24.62 & -11.26 & -9.91 & 16.23 \\
\hline & LC_Random & -38.81 & -45.97 & -50.21 & 13.13 & -7.90 & -5.16 & -8.56 & -23.61 & -30.44 & 24.87 \\
\hline
\end{tabular}




\section{Conclusions}

In this paper we gave a comprehensive survey of aggregation techniques for energy problems presented in the literature. We grouped them into three main categories, and selected approaches from each category such that different complexity levels of the aggregations were covered. Furthermore, we introduced a number of new techniques within each of the categories. This resulted in a selection of ten different aggregation strategies, which were applied to three different data sets, and for three different element types. Furthermore, four of the ten strategies were categorized as Grouping Strategies (GSs) and hence combined with five different selection criteria. This resulted in 219 different aggregations that were constructed and validated according to their ability to replicate the original time series and the ability of the aggregated problem to replicate investments found by solving the original problem.

Comparing the performance of the aggregated problems to the original problems showed, that none of the aggregated problems found the optimal investments. Similarly to previous studies, our results showed that aggregated problems tend to underestimate flexibility and overestimate total system costs. Still, in many cases very good solution quality was achieved, especially for day and week selections. Here $76 \%$ of the solutions only deviated $0.01 \%$ from the original solution when looking at the objective value. For other strategies deviations of up to $3 \%$ were seen. Generally, it can be concluded that properly selected aggregation techniques can achieve high quality solutions with up to $98 \%$ reduction in solution times.

We also investigated whether some aggregation techniques performed better than others and whether more complex aggregation strategies lead to better solutions. The conclusion was that good performance could be observed for both very simple aggregation techniques as well as for complex techniques. The overall best performing strategies were the Statistical Representation (SR) and the Level-Correlation clustering (LC) with random selection, where the SR is very simple and the LC is more challenging. Only a single strategy, namely the RLDC Selection (RL), performed significantly worse than the others, which we believe is due to the construction from purely hourly selection, giving the strategy a disadvantage in the comparison. Moreover, including some randomness and accounting for both residual level and correlation in the selection, seem to be good guidelines to develop a well performing aggregation technique.

The consistency of aggregation performance for different element types was tested, showing that aggregations based on day and week selections seem to have good and generally similar performance, while aggregations based on hour selections have significantly worse performance and generally higher solution times. A closer study of the aggregations unveiled, that those based on hour selections tend to overestimate minimum and maximum rampings as well as the total variance, which potentially could be a reason for the worse performance. However, analyzing performance values of the aggregated time series with the goal of finding a correlation between the similarity to the performance values of the original time series and the aggregated problem performance, showed that, despite our variety of both aggregation techniques as well as data series, in general such a correlation does not seem to be present.

A main contributions was to introduce the concepts of data validation and model validation. The former measures the similarity between the original and the aggregated instances. The latter measures the similarity in the resulting investment decisions by the original and the aggregated problems. With the literature indicating a general believe of better aggregated problem performance being seen when the aggregated time series replicates the RLDC of the original time series well [1,23,24], we combined our model performances with such a data validation to test this. From our results, the conclusion was that a correlation between data and model validation was not seen. Good model validation performance could be observed both when the aggregated time series replicated the original data well, and when not.

Finally, the aggregation techniques based on groupings of elements, followed by a selection from each group, were analyzed with the goal of studying whether a single dominating selection criteria could be identified. With five different selection criteria included in our analysis we saw that random selection on average gave the best aggregated problem performance. This was also seen in relation to the similarity 
within the groups, and here we concluded that, in general, higher similarity within groups provides the benefit of the selection criterion having less impact on the performance of the aggregation technique.

Our conclusions are based on studying a carefully constructed energy model covering many technologies, where we tested three years of real-life demand data. To further verify the conclusions of our work, future work is to apply the same methodology to models with different model features. In particular we want to test whether features providing the energy model with more flexibility make it easier for the aggregations to replicate the original solution.

\section{References}

1. IRENA (2017). Planning for the renewable future: Long-term modelling and tools to expand variable renewable power in emerging economies. International Renewable Energy Agency, Abu Dhabit, 2017.

2. EIA Energy Information Administration. The electrivity market module of the national energy modeling. system model dokumentation 2013. EIA, U.S. Department of Energy - Washington, DC, Tech.rep., 2013.

3. Björn Bahl, Alexander Kümpel, Matthias Lampe, and André Bardow. Time-series aggregation for synthesis of distributed energy supply systems by bounding error in operational expenditure. Computer Aided Chemical Engineering, 38:793-798, 2016.

4. Qixin Chen, Chongqing Kang, Qing Xia, and Jin Zhong. Power generation expansion planning model towards lowcarbon economy and its application in china. IEEE Transactions on Power Systems, 25(2):1117-1125, 2010.

5. Fernando J. de Sisternes. Investment model for renewable electricity system (imres): an electricity generation expansion formulation with unit commitment constraints. MIT Center for Energy and Environmental Policy Research, 2013

6. Fernando J. de Sisternes and Mort D Webster. Optimal selection of sample weeks for approximating the net load in generation planning problems. Massachusetts Institute of Technology. Engineering Systems Division, 2013.

7. Fernando J. de Sisternes, Mort D. Webster, and Ignacio J. Pérez-Arriaga. The impact of bidding rules on electricity markets with intermittens renewables. IEEE Transactions on Power Systems, 30(3):1603-1613, 2015.

8. Energinet.dk. Markeds data.

9. Nicola Falcon and Esteban Gil. Appendix g3 capacity expansion planning for the New Zealand electricity market. Report to Transpower- J1520 Draft Report, 2007.

10. Scott Fortmann-Roe. Understanding the bias-variance trade-off, june 2012.

11. Matthias Fripp. Switch: A planning tool for power systems with large shares of intermittent renewable energy. Environmental Science \& Technology, 46(11):6371-6378, 2012.

12. Richard Green, Iain Staffell, and Nicholas Vasilakos. Divide and conquer? k-means clustering of demand data allows rapid and accurate simulations of the british electricity system. IEEE Transactions on engineerring management, 61(2):251-260, 2014.

13. Markus Haller, Sylvie Ludig, and Nico Bauer. Decarbonization scenarios for the EU and MENA power system: Considering spatial distribution and short term dynamics of renewable generation. Energy Policy, 47(30):282-290, 2012.

14. J. A. Hartigan and M. A. Wong. Algorithm as 136: A k-means clustering algorithm. Applied Statistics, 28(1):100-108, 1979.

15. Alboukadel Kassambara. Practical Guide To Cluster Analysis in R - Unsuperviced Machine Learning. STHDA, 2017.

16. Hardi Kudovere, Stefanie Buchholz, and Hans Ravn. Constructing aggregated time seried data for energy system model analysis. DTU-Orbit, Technical Report, 2017.

17. Yixian Liu, Ramteen Sioshansi, and Antonio J. Conejo. Hierarchical clustering to find representative operating periods for capacity-expansion modeling. IEEE Transactions on Power Systems, 33(3):3029-3039, 2017.

18. Sylvie Ludig, Markus Haller, Eva Schmid, and Nico Bauer. Fluctuating renewables in a long-term climate change mitigation strategy. Energy, 36(43):6674-6685, 2011.

19. Christoffer Ernst Lythcke-Jørgensen, Marie Münster, Adriano Viana Ensinas, and Fredrik Haglind. A method for aggregating external operating conditions in multi-generation system optimization models. Applied Energy, 166(5):59$75,2016$.

20. M. Salama M. ElNozah and and R. Seethapathy. A probabilistic load modelling approach using clustering algorithms. IEEE Power and Energy Society General Meeting, pages 1-5, 2013.

21. James H. Merrick. On representation of temporal variability in electricity capacity planning models. Energy Economics, 59(19):261-274, 2016.

22. Francisco D. Munoz and Andrew D. Mills. Endogenous assessment of the capacity value of solar pv in generation investment planning studies. Transactions on Sustainable Energy, 6(4):1574-1585, 2015.

23. Paul Nahmmacher, Eva Schmid, Lion Hirth, and Brigitte Knopf. Carpe diem: A novel approach to select representative days for long-term power system models with high shares of renewable energy sources. Energy, 112(39):430-442, 2016. 
24. Marco Nicolos, Andrew Mills, and Ryan Wiser. The importance of high temporal resolution in modeling renewable energy penetration scenarios. 9th Conference on Applied Infrastructure Research, TU Berlin, Berlin, Germany, October 8-9, 2010, 2011

25. C. I. Nweke, F. Leanez, G. R. Drayton, and M. Kolhe. Benefits of chronological optimization in capacity planning for electricity markets. IEEE Power System Technology, Conference paper (POWERCON 2012), 2012.

26. Ministry of Foreign Affairs of Denmark. Independent from fossil fuels by 2050.

27. Kris Poncelet, Erik Delarue, Daan Six, Jan Dueinck, and William D'haeseleer. Impact of the level of temporal and operational detail in energy-system planning models. Applied Energy, 162(58):631-643, 2015.

28. Kris Poncelet, Hanspeter Hoschle, Erik Delarue, Ana Virag, and William D'haeseleer. Selecting representative days for capturing the implications of integrating intermittent renewables in generation expansion problems. IEEE Transactions on Power Systems, PP(99):1-1, 2016.

29. David F. Rogers, Robert D. Plante, Richard T. Wong, and James R. Evans. Aggregation and disaggregation techniques and methodology in optimization. Operations Research, 39(4):553-582, 1991.

30. Sheila Samsatli and Nouri J. Samsatli. A general spatio-temporal model of energy systems with a detailed account of transport and storage. Computers and Chemical Engineering, 80(13):155-176, 2015.

31. Walter Short, Patrick Sullivan, Trieu Mai, Matthew Mowers, Caroline Uriarte, Nate Blair, Donna Heimiller, and Andrew Martinez. Regional energy deployment system (reeds). NREL, Tech.rep., 2011.

32. Falko Ueckerdt, Robert Brecha, and Gunnar Luderer. Analyzing major challenges of wind and solar variability in power systems. Renewable Energy, 81(1):1-10, 2015.

33. Falko Ueckerdt, Robert Brecha, Gunnar Luderer, Patrick Sullivan, Eva Schmid, Nico Bauer, Diana Böttger, and Robert Pietzcker. Representing power sector variability and the integration of variable renewables in long-term energy-economy models using residual load duration curves. Energy, 90, part 2(56):1799-1814, 2015. 


\section{A Terminology}

\section{Nomenclature}

$\begin{array}{ll}A T & \text { Aggregation Technique } \\ B S & \text { Benchmark Solution } \\ C A & \text { Cluster Analysis } \\ C C & \text { Cluster Cluster } \\ C C G T & \text { Combined Cycle Gas Turbine } \\ C D C & \text { Correlation Duration Curve } \\ C E M U C & \text { Capacity Expansion Model with Unit Commitment } \\ D B & \text { Dynamic Blocking } \\ D C & \text { Duration Curve } \\ D X & \text { Dummy selection } \\ E S & \text { Exhaustive Search } \\ G S & \text { Grouping Strategies } \\ H C & \text { Hieracical Clustering } \\ H S & \text { Heuristic Selection } \\ I S & \text { Investment Selection } \\ L C & \text { Level-Correlation Cluster } \\ L D C & \text { Load Duration Curve } \\ M I L P & \text { Mixed Integer Linear Program } \\ N G S & \text { Non-Grouping Strategies } \\ N R M S E & \text { Normalized Root Mean Square Error } \\ O A & \text { Optimized RLDC Approximation } \\ O C G T & \text { Open Cycle Gas Turbine } \\ O P T & \text { Optimization } \\ O S & \text { Optimal criteria Selection } \\ P C & \text { Partitioning Clustering } \\ P I & \text { Performance Index } \\ P V & \text { Photpvoltaics } \\ R D C & \text { Ramping Duration Curve } \\ R L & \text { RLDC Selection } \\ R L C & \text { Residual Load Curve } \\ R L D C & \text { Residual Load Duration Curve } \\ S C & \text { Single Cluster } \\ S R & \text { Statistical Representation } \\ U C & \text { Unit Commitment } \\ V R E & \text { Variable Renewable Energy } \\ & \end{array}$




\section{B Supplements to the Literature Review}

Table 21: Articles considering aggregated time series based on Heuristic Selection

\begin{tabular}{|c|c|l|}
\hline \multicolumn{1}{|c|}{ Article } & \# Time slices & \multicolumn{1}{l|}{ Chosen according to } \\
\hline$[11]$ & 576 & A peak-load day and a randomly chosen day for each month, each day in 24h resolution \\
{$[24]$} & 288 & 3 days for each of the 4 seasons, 1 workday and both weekend days, each day in $24 h$ resolution \\
{$[21]$} & 144 & A peak-load day and a median day for each month, each day having 4h resolution \\
{$[31]$} & 17 & 1 day for each of the 4 seasons (6h resolution), additional slice representing summer-peak \\
{$[24]$} & 16 & 2 days for each of the 4 seasons, each day divided into a night and a peak slice \\
{$[2]$} & 9 & 3 seasons (summer, winter, spring/fall) represented by a peak, intermediate and base slice \\
{$[3]$} & $1-10$ & Not specified in the paper \\
\hline
\end{tabular}

Table 22: Articles considering aggregated time series based on Cluster Analysis

\begin{tabular}{|c|c|l|l|}
\hline Article & \# Clusters & Cluster technique & \multicolumn{2}{l|}{ Chosen according to } \\
\hline$[22]$ & 83 (42 when modified) (1992 and 1008 time slices) & $k$-mean cluster \& Peak-load modification & Centroid of each cluster \\
{$[17]$} & 10, each clustered into $3(720$ time slices $)$ & Hiearical, $k$-means and combination & Unknown \\
{$[12]$} & $10(240$ time slices) & $k$-mean cluster & Average day (demand level and hour-by-hour changes in demand) \\
{$[20]$} & $6(144$ time slices $)$ & Hiearical, Fuzzy and $k$-means & Mean and Median day \\
{$[23]$} & $6(48$ time slices) & Hiearical cluster & Day closest to the centroid \\
{$[19]$} & $46(46$ time slices) & $k$-mean cluster & Weighted average of cluster elements \\
\hline
\end{tabular}

Table 23: Summary of the literature articles and their validation method. AT denotes Aggregation Technique

\begin{tabular}{|c|c|l|l|l|}
\hline Article & AT & \multicolumn{2}{c|}{ Model } \\
\hline$[30]$ & HS & Spatio-temporal MILP model optimal network structure and operation & No & Model Validation \\
{$[24]$} & HS & THEA, linear optimization dispatch and investment model & Yes (Graphical illustration) & No (Intractable model) \\
{$[18]$} & HS & LIMES - Long-term Investment Model & No & Yes (Original model) \\
{$[13]$} & HS & LIMES-EU & No (A maximum of 4 days is included, Resolution test) \\
{$[12]$} & CA & Simple dispatch model that estimates grid operation patterns and costs & No & No \\
{$[21]$} & CA & Capacity planning models - investments and operation & No (But variance cover for different amount of clusters) & Yes (Original model) \\
{$[23]$} & CA & LIMES (Original model) \\
{$[22]$} & CA & Deterministic capacity expansion model & Yes & No (A maximum of 100 days is included, Resolution test) \\
{$[19]$} & CA & Simple operation optimization model of a CHP plant & No & Yes (Original model compare) \\
{$[3]$} & CA & Investment model & No & Yes (Original model compare) \\
{$[28]$} & OPT & LUSYM - investment model & No & Yes (Compared to approximation of original solution) \\
{$[33]$} & OPT & REMIN-D investment model for Germany & Yes & Yes (Original model) \\
{$[6]$} & OPT & Capacity planning models & No & Yo \\
{$[7]$} & OPT & IMRES a centralized capacity expansion model & Yes & No \\
\hline
\end{tabular}

\section{Supplements to the Test cases results}


Table 24: The 20 different solutions achieved for the 219 single aggregations

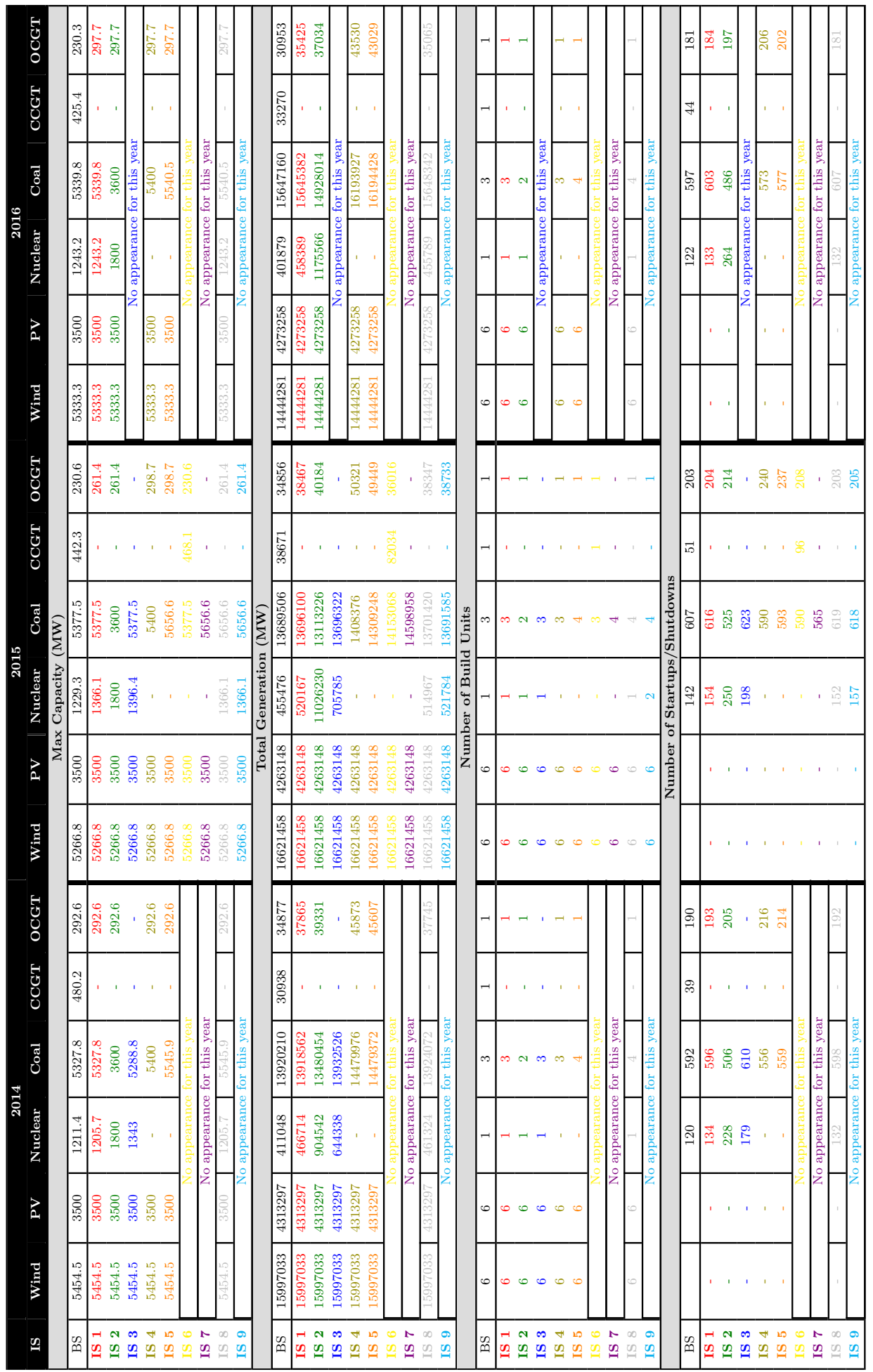




\section{Aggregation Performance}

Table 25: Amount of different ISs observed within each type of grouping

\begin{tabular}{|c|c|c|c|c|c|c|c|c|c|}
\hline Strategy & Hour & $\begin{array}{l}2014 \\
\text { Day }\end{array}$ & Week & Hour & $\begin{array}{l}2015 \\
\text { Day }\end{array}$ & Week & Hour & $\begin{array}{l}2016 \\
\text { Day }\end{array}$ & Week \\
\hline DB & 2 & 3 & 4 & 1 & 3 & 3 & 1 & 2 & 2 \\
\hline $\mathrm{SC}$ & 2 & 2 & 3 & 2 & 2 & 2 & 2 & 2 & 2 \\
\hline $\mathrm{CC}$ & 1 & 1 & 2 & 1 & 2 & 2 & 2 & 1 & 3 \\
\hline $\mathrm{LC}$ & 2 & 2 & 2 & 2 & 2 & 2 & 3 & 3 & 2 \\
\hline
\end{tabular}

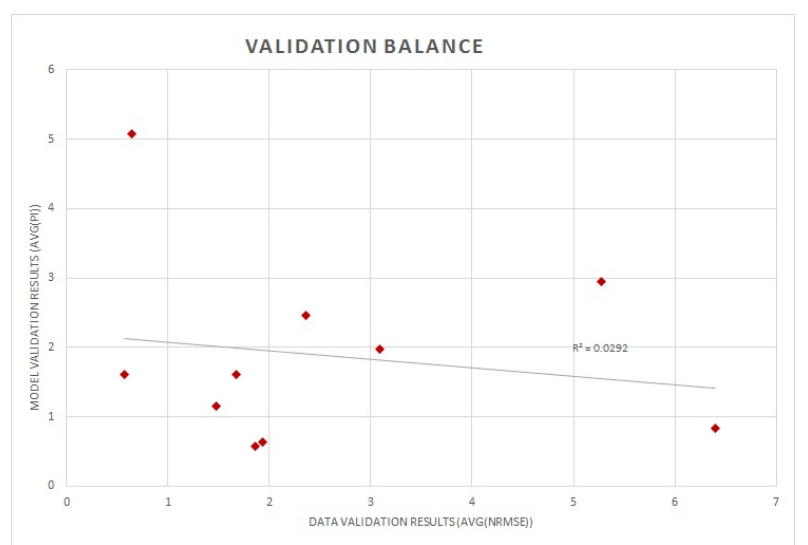

Fig. 7: Relation between average NRMSE and average PI for all strategies. Averages are over all elements and years

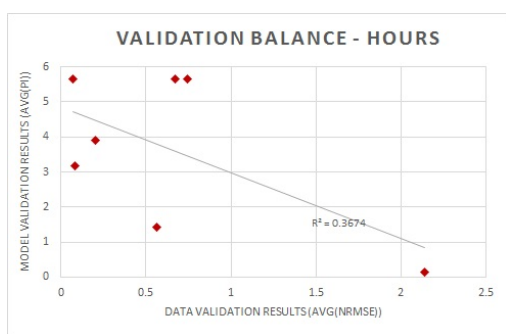

(a) Hours

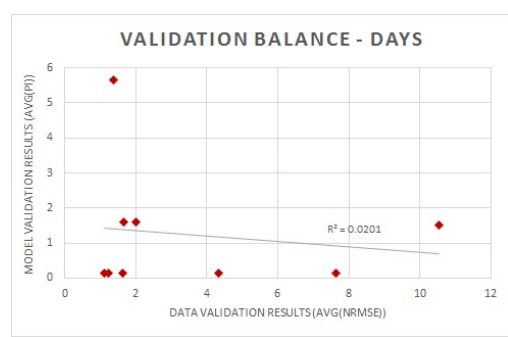

(b) Days

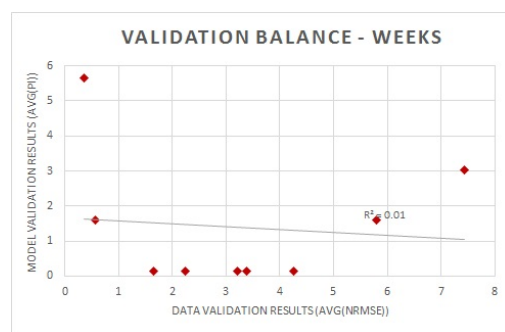

(c) Weeks

Fig. 8: Relation between yearly averaged NRMSE and yearly averaged PI for all strategies devided into hour, days, and week elements 
Table 26: Solution times in seconds for non-aggregated problems with fixed investment choices found by solving the aggregated problems

\begin{tabular}{|c|c|c|c|c|c|c|c|c|c|c|c|c|c|}
\hline \multirow[b]{2}{*}{ Strategy } & \multicolumn{4}{|c|}{ Hour } & \multicolumn{4}{|c|}{ Day } & \multicolumn{4}{|c|}{ Week } & \multirow[b]{2}{*}{ AVG Time } \\
\hline & 2014 & 2015 & 2016 & AVG & 2014 & 2015 & 2016 & AVG & 2014 & 2015 & 2016 & AVG & \\
\hline DX & 140.16 & 227.22 & 156.39 & 174.59 & 67.33 & 93.76 & 135.78 & 98.96 & 89.13 & 206.42 & 134.02 & 143.19 & 138.91 \\
\hline SR & 76.23 & 191.92 & 158.59 & 142.25 & 297.98 & 133.25 & 109.38 & 180.20 & 53.51 & 228.29 & 74.17 & 118.66 & 147.04 \\
\hline $\mathrm{RL}$ & 199.33 & 166.14 & 156.85 & 174.11 & 127.81 & 170.21 & 155.75 & 151.26 & 182.42 & 152.13 & 157.99 & 164.18 & 163.18 \\
\hline ES & - & - & - & - & - & - & - & - & 95.55 & 229.01 & 132.4 & 152.32 & 152.32 \\
\hline $\mathrm{OA}$ & - & - & - & - & 97.80 & 172.81 & 121.46 & 130.69 & - & - & - & - & 130.69 \\
\hline OS & - & - & - & - & 53.94 & 137.4 & 153.58 & 114.97 & 66.22 & 179.95 & 233.80 & 159.99 & 137.48 \\
\hline DB_Random & 121.19 & 153.47 & 171.07 & 148.58 & 83.53 & 116.11 & 96.38 & 98.67 & 109.04 & 82.76 & 142.73 & 111.51 & 119.59 \\
\hline SC_Random & 110.66 & 288.44 & 230.11 & 209.73 & 85.83 & 169.76 & 121.02 & 125.54 & 107.9 & 182.75 & 203.9 & 164.85 & 166.71 \\
\hline CC_Random & 181.08 & 135.73 & 165.23 & 160.68 & 111.67 & 136.74 & 119.31 & 122.57 & 52.3 & 58.38 & 77.23 & 62.64 & 115.30 \\
\hline LC_Random & 111.24 & 215.88 & 251.58 & 192.9 & 204.35 & 153.02 & 130.24 & 162.54 & 96.78 & 102.27 & 124.62 & 107.89 & 154.44 \\
\hline
\end{tabular}

